\title{
ELASTIC-PLASTIC MODELLING OF SHAPED CHARGE JET PENETRATION
}

\author{
By Roman Novokshanov And John OCKEndon \\ Oxford Centre for Industrial and Applied Mathematics, Mathematical Institute, \\ University of Oxford, 24-29 St Giles', Oxford OX1 3LB, UK
}

This paper concerns the mathematical modelling of high rate penetration of a metal target by a shaped charge device that produces a high velocity jet. A key objective is to predict the penetration velocity, be it subsonic, transonic, or supersonic. We do this by considering, on the local scale near the tip of the penetrated cavity, an elastic-plastic free boundary problem that takes into account the residual stresses produced by the moving plasticized region of the target. It is the self-consistency of this elastic-plastic model that dictates predictions for the penetration velocity.

Keywords: penetration, shaped charge, free boundary

\section{Introduction}

Shaped charges are of great importance in many industrial applications, ranging from oil recovery to defence. The basic physical mechanism is the penetration of a solid target by a high velocity jet which is created by the very rapid plasticization of a liner in a shaped charge device. In this work we will simply assume that the liner is transformed into a high velocity planar or cylindrical jet that can be modelled as continuous inviscid liquid. It is the interaction between this jet and the target that dictates the penetration velocity.

We will give a more detailed overview of the physical evidence concerning the penetration of metal targets in $\S 2$, but the salient large scale features are illustrated in figures 1-2 (courtesy of Dr J. Curtis and Dr C. Poole). Here a cylindrical hole or 'cavity' has been produced by a jet of radius around $2 \mathrm{~mm}$, moving at about 10 $\mathrm{km} / \mathrm{s}$.

The most striking observations are that

- the cavity is nearly cylindrical away from the boundaries of the target and its radius is comparable to that of the jet;

- the target has deformed appreciably at distances of many radii from the cavity. This deformation takes the shape of bulging of the boundary where it is closest to the cavity, the volume of the bulging being comparable to the cavity volume.

During the experiments, heating of only a few hundred ${ }^{\circ} \mathrm{C}$ was reported immediately after penetration. 


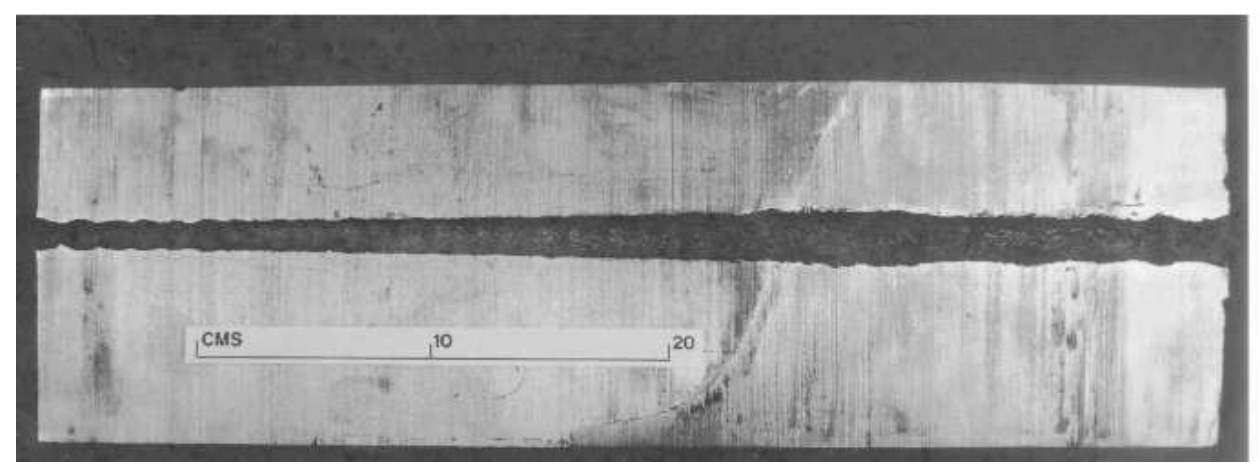

Figure 1. Penetrated target; penetration from right to left.

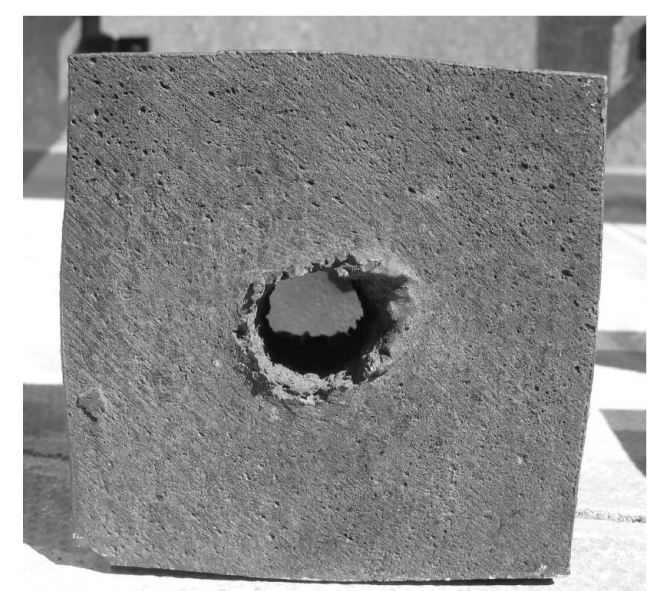

Figure 2. Entry into a penetrated target $(10 \mathrm{~cm} \times 10 \mathrm{~cm})$.

There are many less clear-cut observations concerning the metallurgy of the penetrated target that will be listed in $\S 2$, which will also contain a brief review of the theoretical literature.

Our objective is to construct, for metal targets, the simplest possible systematic macroscopic mathematical model that is consistent with this evidence. To do this we will make use of several theories from solid and fluid mechanics. Our starting point is to regard the target as a solid which is initially a homogeneous, unstressed, isotropic elastic material of infinite extent. We regard the jet impact as simply providing a localized high pressure that is applied to the target just in the cavity tip or 'crater' region, whose dimensions are comparable to those of the jet and cavity; the static pressure in the jet may be as high as $100 \sigma_{Y}$, where $\sigma_{Y}$ is the yield stress of the target, but the pressure in the rebounding jet in the cavity will be neglected.

Despite the high pressures involved, the basic piece of modelling from which our theory starts is the theory for a purely elastic target subject to a localized unidirectional force at a crater which leaves behind a low pressure cavity in its wake; here and henceforth we will use the word cavity to describe the penetrated region of the target behind the crater, even though part of this region is occupied 
by the jet. We will carry out this calculation in $\S 3$ using the methodology of scalar stress functions, both in the usual case of an axisymmetric shaped charge and in the less common case of a two-dimensional planar or 'linear' shaped charge. Although there is a stark contrast between this elastic problem and the classical theory of dynamic brittle fracture, we will make use of the common idea of representing the cavity and crater as distributions of singular solutions of the equations of linear elasticity. However we emphasize that, for shaped charges, penetration is driven by a locally applied force in a geometry which is usually axisymmetric, and that the crater speed may well be supersonic.

This preliminary modelling will enable us to estimate the extent to which a large target is plasticized in a travelling-wave situation in which the crater advances with constant speed. To do this we first plot the stress contours relevant to the Von Mises yield criterion for the elastic response just to a point force at the tip of a prescribed cavity. Then, in $\S 3$ we use this as the first step of an iterative procedure to estimate the extent of the plastic region. To make this iteration realistic we must at each stage incorporate an updated estimate of the extent of the region of residual stress that is left behind the propagating plastic region, as shown schematically in figure 3. At each stage of the iteration, the reference stress in this region is that which the material remembers from the instant at which it left the plastic zone.

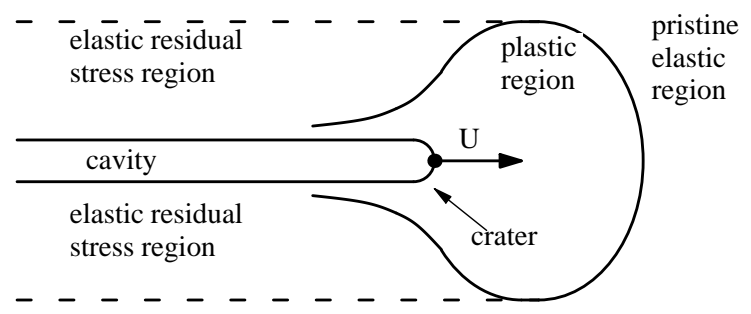

Figure 3. Elastic-plastic shaped charge penetration.

The output of our iterative procedure is an approximate solution of the complicated free boundary problem of figure 3, where the boundaries between the plastic and the pristine and residually stressed elastic regions are unknown in advance. It is the plastic region which dictates the penetration velocity and this region is acted on by both a system of elastic tractions and the crater pressure. However, we will find that when the jet pressure is so high that the plastic region is much larger than the cavity radius, which can easily happen in practice, we can follow the idea of Hill et al. (1944) and represent this region, like the jet, as an inviscid liquid. In this case the free boundary problem for the elastic-plastic boundaries can be approximated by a Helmholtz flow and it is the force balance in this Helmholtz flow that determines the penetration velocity. The lowest order approximation to this velocity turns out to be that predicted by Birkhoff et al. (1948). However, by working in the scenario of figure 3 we will be able to both assess the conditions under which the formula in Birkhoff et al. (1948) is realistic and to suggest how it could be made more accurate.

Although we will only consider travelling-wave models in an infinite target, there is no reason in principle why our theoretical framework should not apply to 
Table 1. Material parameters

\begin{aligned} & \hline jet velocity $2.5-10 \mathrm{~km} / \mathrm{s} \\ &$ jet radius $2-4 \mathrm{~mm} \\ &$ jet density (copper) $\rho_{j} \sim 8.9 \times 10^{3} \mathrm{~kg} / \mathrm{m}^{3} \\ &$ yield stress (steel) $\sigma_{Y} \sim 2 \times 10^{9} \mathrm{~kg} / \mathrm{ms}^{2} \sim 2 \mathrm{GPa} \\ &$ shear modulus (steel) $\mu \sim 0.8 \times 10^{11} \mathrm{~kg} / \mathrm{ms}^{2} \sim 80 \mathrm{GPa} \\ &$ bulk modulus (steel) $K \sim 1.4 \times 10^{11} \mathrm{~kg} / \mathrm{ms}^{2} \sim 140 \mathrm{GPa} \\ &$ Lame constant (steel) $\lambda=K-2 \mu / 3 \sim 1 \times 10^{11} \mathrm{~kg} / \mathrm{ms}^{2} \sim 110 \mathrm{GPa} \\ &$ density (steel) $\rho_{t} \sim 9 \times 10^{3} \mathrm{~kg} / \mathrm{m}^{3} \\ &$ penetration depth $0.3-1 \mathrm{~m} \\ &$ cavity, crater radius $a \sim 7.5-15 \mathrm{~mm} \\ &$ S-wave speed (steel) $c_{s}=\sqrt{\mu / \rho} \sim 3 \mathrm{~km} / \mathrm{s} \\ &$ P-wave speed (steel) $c_{p}=\sqrt{(\lambda+2 \mu) / \rho} \sim 5.4 \mathrm{~km} / \mathrm{s} \\ &$ Poisson ratio $\nu=\lambda /(2(\lambda+\mu)) \sim 0.28 \\ &$ sound speed ratio $\gamma=c_{p} / c_{s} \sim 1.8 \\ &$\hline\end{aligned}

decelerating penetration in a finite target so as to give a prediction for penetration distances.

\section{Experimental and theoretical background}

(a) Experimental background

Table 1 gives a rough idea of the parameter ranges that are typical for high velocity penetration. They apply to the case of a copper jet penetrating a target of rolled homogeneous armour, which is a high strength steel (Barnea \& Sela 1996; Walters \& Zukas 1989; Walker 2001). We remark that, since the static jet pressure $\sim 20-100 \mathrm{GPa}$, shaped charge penetration is fundamentally different from water jet penetration (Eddingfield et al. 1981; Swanson et al. 1987; Watson \& Moxon 1985). Indeed, it has more in common with rod penetration (Ravid et al. 1995; Tate 1986a, b; Walker \& Anderson 1995). Theories for more general solid penetrators are reviewed in Backman \& Goldsmith (1978).

Most experimental evidence comes from post facto metallurgical analysis, but Cook (1959) provides direct experimental observations of several ultra-high velocity impacts of Lucite blocks by shaped charges with steel charge jets. There is clear visual evidence of the supersonic nature of penetration in a solid, which results in the appearance of shock waves behind the propagating crater. Further evidence concerning shock waves is given in Murr et al. (1996, 1997), which analyses the microstructure of copper targets after hypervelocity penetration by a copper charge jet. Microbands were observed in a cylindrical region around the cavity, these bands being induced by shear from the shock waves produced by the crater. Although no significant melting was apparent, there was evidence of dynamic recrystallization in a narrow region around the cavity, and the target material 'jetted' into this region. Similar damage has been observed in hypervelocity projectile impact (Duan et al. 2003; Wells et al. 2002).

The penetration of stacked high-strength steel plates by a copper jet has been investigated in Yin et al. (2004). The microstructure of the 'etching' layer around the cavity, which is around $100 \mu \mathrm{m}$ thick, is a mixture of martensite and austenite, both of nanometer scale. The existence of recrystallized austenite grains in this 
layer was attributed to localized heating up to $1000^{\circ} \mathrm{C}$. Whereas in Yin et al. (2004) there was evidence of local softening in the etching layer, the measurements of Poole (2005) revealed greater local hardness in the case of high strength steel penetrated by a silver jet. Many microcracks were observed in a layer $1 \mathrm{~mm}$ thick around the cavity, these microcracks often containing jetted silver.

Even this brief review reveals that microstructural analysis does not yet allow obvious deductions to be made concerning the macroscopic modelling of the target during penetration. In particular, there is no clear evidence to suggest the importance of energy transfer as compared to momentum transfer; this question has been considered in Walker (2001) and we will return to it in the conclusion. However, plastic flow and fracture certainly occur and, in view of figures 1-2, we will assume that the former is prevalent except in a thin region around the cavity.

\section{(b) Theoretical background}

The very first models of shaped charge penetration (Birkhoff et al. 1948; Birkhoff \& Zarantonello 1957) assumed that the target and jet were so highly plasticized that they could both be modelled as inviscid incompressible fluids.

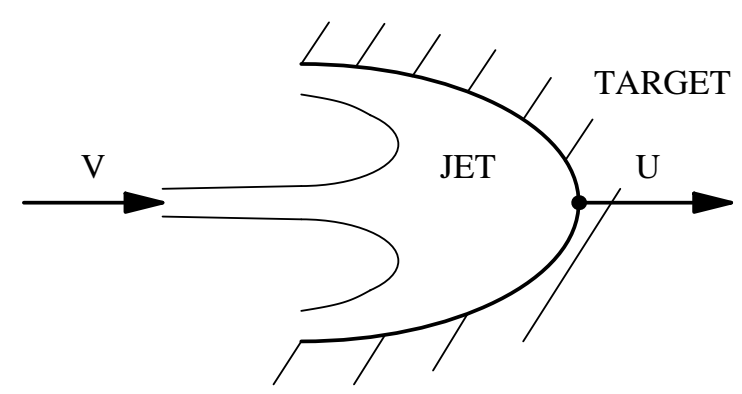

Figure 4. Crater mechanics for the hydrodynamic model.

Assuming negligible stresses far from the crater and, using the scenario of figure 4 in a frame moving with the crater velocity $U$, Bernoulli's equation for the pressures in the jet and target at the stagnation point immediately implies that

$$
\frac{1}{2} \rho_{j}(V-U)^{2}=\frac{1}{2} \rho_{t} U^{2}
$$

where $\rho_{j}, \rho_{t}$ are the jet and target densities and $V$ is the jet velocity. Hence the penetration velocity $U$ is simply

$$
U=\frac{V}{1+\sqrt{\rho_{t} / \rho_{j}}} .
$$

Since, in the moving frame, the velocity of the jet is $V-U$, the time required for a jet of length $L$ to penetrate is just $L /(V-U)$. Consequently, the depth of penetration $P$ is

$$
P=L \sqrt{\rho_{j} / \rho_{t}}
$$

A more elaborate model for $P$ in which penetration continued after the jet ceased to exert any pressure on the target was proposed in Pack \& Evans (1951). In the 
case of two-dimensional penetration, the hydrodynamic model may be incompatible with the existence of a cavity of uniform thickness; indeed, when $\rho_{j}=\rho_{t}$, the shape of the jet/target boundary can be found by conformal mapping as in Birkhoff \& Zarantonello (1957), and it is asymptotically parabolic far from the crater. This defect was resolved in an ad hoc way in Hopkins \& Robertson (1967).

An early proposal to account for a non-negligible target yield stress was made in Eichelberger \& Pugh (1952). Based on the idea that target strength was important in the later stages of penetration, (2.1) was modified to

$$
\frac{1}{2} \rho_{j}(V-U)^{2}+\sigma_{j}=\frac{1}{2} \rho_{t} U^{2}+\sigma_{t},
$$

where $\sigma_{j}$ and $\sigma_{t}$ are measures of the resistances of the jet and the target to the plastic deformation. This idea was further extended in Szendrei $(1995,1998)$. However the first systematic study of the confining effect of the target strength was made relatively recently in Tate $(1986 a, b)$. These papers focused on the axisymmetric elastic-plastic response of the target far downstream of the cavity. The Von Mises criterion was used in conjunction with the assumption of perfect plasticity in order to estimate the parameters in (2.4). In Barnea \& Sela (1996), a time-dependent 'cavity expansion' model was derived using similar physical assumptions. This led to an ordinary differential equation for the cavity radius in the vicinity of the crater as a function of time, which is considered to be equivalent to distance downstream of the crater. However, this approach, like that of Szendrei (1995, 1998), suffers from the presence of undetermined constants which need to be obtained by matching with a solution in the crater region. A further refinement was made in Poole (2005) where account was taken of the residual stresses left behind when the cavity expansion pressure was released to atmospheric.

In summary, although substantial theoretical target penetration research has been carried out using both fluid and solid mechanics models, there is at present only a rather tenuous link between these models via equations like (2.4). It is one of our aims in this paper to make this connection more systematic.

We have not mentioned any of the numerous purely numerical models for penetration such as hydrocodes (Cornish 2001; Huntington-Thresher et al. 2001). It is almost inevitable that such codes rely on submodels of the type described above coupled with general continuum mechanics solvers and therefore a systematic target mechanics scenario should also shed light on the efficacy of such codes.

\section{Theoretical framework}

Throughout this section we will continue to assume that the target extends to infinity in all directions and that the jet velocity $V$ and crater velocity $U$ are independent of time. These assumptions will enable us to construct a theoretical framework based on the scenario of figure 3 .

We must bear in mind throughout that it is our principal objective to predict $U$ for a given $V$, and that even though $V$ may easily exceed the sound speeds in the pristine target, the penetration velocity $U$ may still be 'subsonic'. 


\section{(a) Elasticity modelling}

We start regarding the target as linearly elastic, with moduli $\lambda, \mu$, when we are sufficiently far from the crater. Thus, in the far field of the pristine region in figure 3, the target responds to the sum of a force localized around the crater and a suitable distribution of tractions exerted on the boundary of the residual stress region downstream of the crater. We consider these two contributions separately.

\section{(i) Point force response}

The force is the axial resolvent of the traction exerted on that part of the pristine elastic/plastic boundary whose normal has a positive component along the axis of symmetry, i.e. to the right of the dashed lines in figure 3 . In a frame moving with the crater, the elastic response to a point force is obtained by solving the equations of motion with an appropriate delta-function on the right-hand side. As described in the appendix (please note that the appendix is provided as electronic supplementary material), the result can most conveniently be described in terms of a Love stress function $L$ depending on the geometry and the Mach numbers $M_{s}=U / c_{s}, M_{p}=U / c_{p}$, where $c_{s}, c_{p}$ are the $S$ - and $P$-wave speeds.

In all cases a force of strength $F$ is applied at a point on the jet axis moving with speed $U$. We denote this point by $x=U t, y=0$ in two dimensions and by $z=U t, r=0$ in three dimensions with axial symmetry. Despite the slight ambiguity we denote both $x-U t$ and $z-U t$ by $\xi$, the downstream region being $\xi<0$. The relevant Love stress functions are derived in the appendix and displayed as the boxed equations. Also the corresponding $S$ - and $P$ - wave fronts emitted by the moving point force are shown schematically in figure 5 ; the patterns are same for both linear and axisymmetric penetration.

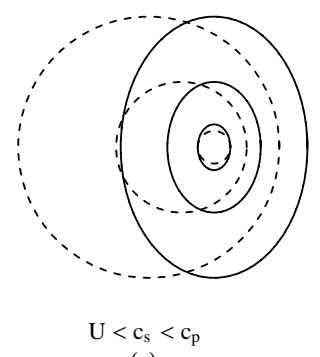

(a)

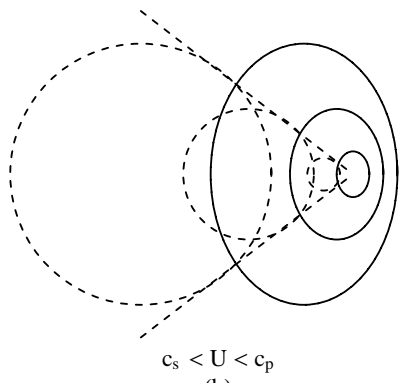

(b)

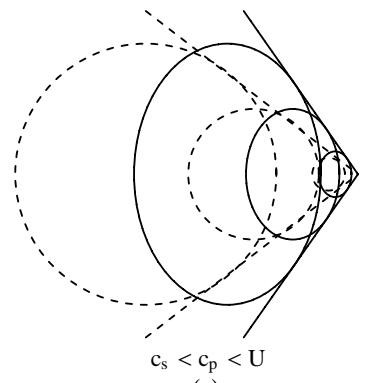

Figure 5. Wavefronts from a point force in (a) subsonic, (b) transonic and (c) supersonic penetration; the P-wavefronts are continuous and the S-wavefronts are dashed.

For illustrative purposes, we have chosen a value of $F$ corresponding to a pressure exerted on the target of $100 \sigma_{Y}$ distributed over the cross-sectional area of the cavity, which is $2 a$ or $\pi a^{2}$ for linear and axisymmetric penetration respectively. Here and subsequently we take $a=7.5 \mathrm{~mm}$. The pressure may of course be much less than $100 \sigma_{Y}$ if the crater velocity is comparable to the sound speeds in the target. Also, for definiteness the Von Mises yield criterion is the one with which we will work in this paper. The results of the appendix allow us to plot the level curves of the Von 
Mises stress intensity $\sigma_{V M}$, namely

$$
\sigma_{V M}=\frac{1}{2} \sqrt{\left(\sigma_{\xi \xi}-\sigma_{y y}\right)^{2}+4 \sigma_{\xi y}^{2}}
$$

in two dimensions, and

$$
\sigma_{V M}=\sqrt{\sigma_{r r}^{2}+3 \sigma_{r \xi^{2}}+\sigma_{\xi \xi^{2}}-\sigma_{\xi \xi} \sigma_{\theta \theta}+\sigma_{\theta \theta^{2}}-\sigma_{r r}\left(\sigma_{\xi \xi}+\sigma_{\theta \theta}\right)}
$$

in three dimensions with axial symmetry. In the quasistatic case $U=0$, the results are given in figure 6 .

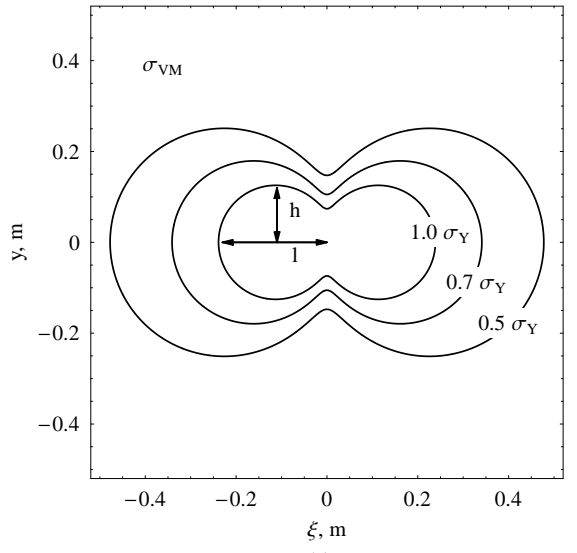

(a)

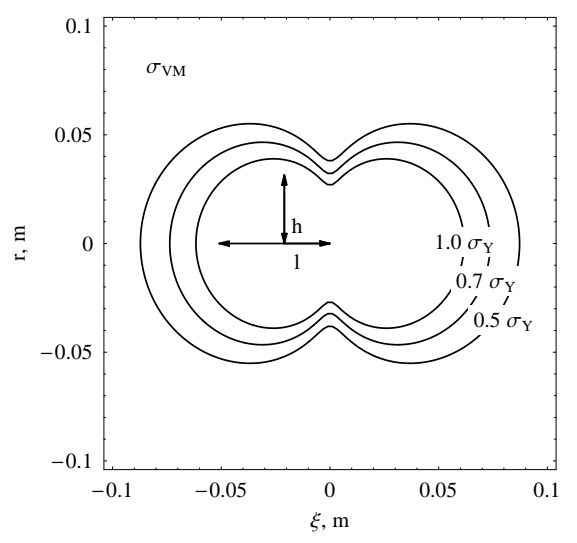

(b)

Figure 6. Von Mises yield contours $\sigma_{V M}$ for a pressure $100 \sigma_{Y}$ corresponding to a force $F=100 \sigma_{Y} \times 2 a$ at $\xi=y=0$ for (a) two dimensions and to a force $F=100 \sigma_{Y} \times \pi a^{2}$ at $\xi=r=0$ for (b) axial symmetry; $h, l$ are dimensionless lengths defined in the text.

In the two-dimensional case, figure 7 shows the dependence of the Von Mises yield criterion level contours, as calculated in the boxed equations in the appendix, on the crater Mach number $U / c_{s}$. We note that in the transonic and supersonic cases, the presence of jump discontinuities in the second derivatives of $L_{F}$ implies that there are delta functions in the stresses along the straight Mach lines in figures $7 \mathrm{~b}-\mathrm{d}$. Indeed, for two-dimensional penetration with $U>c_{p}$, Huygens principle ensures that it is only on these lines that the point force generates any elastic stress. The two transonic plots, figures $7 \mathrm{~b}$, c, reveal that the geometry of the stress contours depends nonmonotonically on the crater velocity. In particular, the length and the breadth of the smooth components of the contours in figure 7 , which will always be of $O\left(F / \sigma_{Y}\right)$, can be represented as $l\left(M_{s}\right)\left(F / \sigma_{Y}\right)$ and $h\left(M_{s}\right)\left(F / \sigma_{Y}\right)$ respectively, where $l, h$ are plotted in figure $8 \mathrm{a}$. We note that the dimensions of the smooth part of the yield contour increase abruptly at each of the sonic 'barriers'. The corresponding results for axisymmetrical penetration are shown in figure 9 and figure $8 \mathrm{~b}$ respectively, where now the length and breadth of the smooth components are $l\left(M_{s}\right)\left(F / \sigma_{Y}\right)^{1 / 2}$ and $h\left(M_{s}\right)\left(F / \sigma_{Y}\right)^{1 / 2}$. It is remarkable that the length is so insensitive to the Mach number in comparison with the two-dimensional case. 


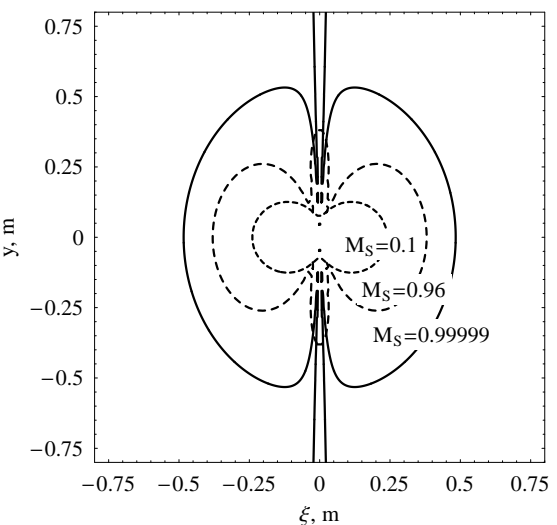

(a)

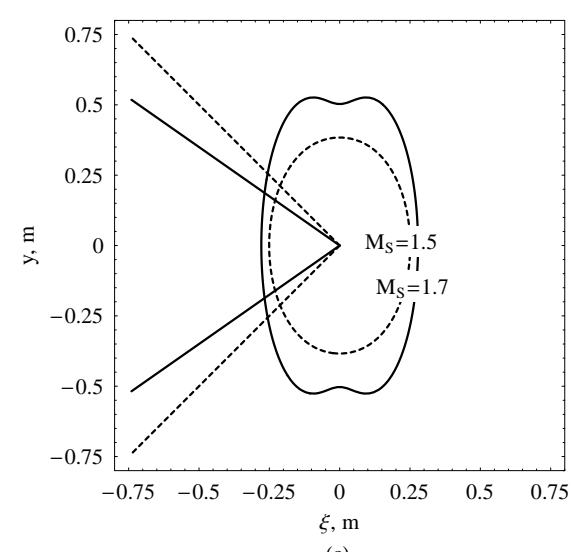

(c)

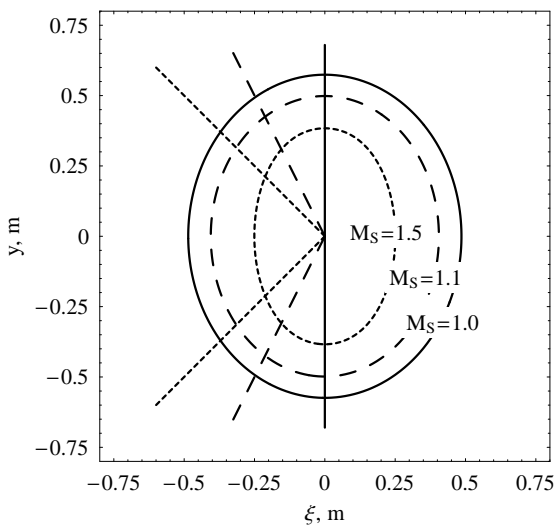

(b)

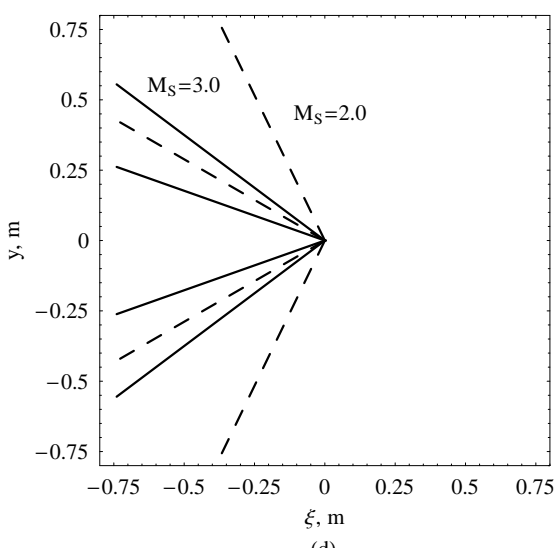

(d)

Figure 7. Dependence of the Von Mises criterion level contours $\sigma_{V M}=\sigma_{Y}$ on the Mach number $M_{s}=U / c_{s}$ for linear penetration using data of Table 1 and the value of $F$ used in figure 6; see text for explanation of the straight lines. Curves (a) correspond to subsonic penetration with $0<M_{s}<1$, (b) to transonic penetration with $1<M_{s}<1.5$, (c) to transonic penetration with $1.5<M_{s}<c_{p} / c_{s}$ and (d) to supersonic penetration with $c_{p} / c_{s}<M_{s}$.

\section{(ii) Response to residual stress}

There will inevitably be a region of residual stress in the elastic target downstream of the plasticized region. Were the elastic/plastic boundary to be precisely as in figure 7 , then we could immediately identify this residually stressed region. It would simply comprise the particles that have traversed all rearward-facing segments of the elastic/plastic boundary, as shown schematically in figure 10 for subsonic penetration.

At distances large compared to the thickness of the residual stress region, the stresses created by that region can be represented as distributions of solutions of the equations of pristine linear elasticity that have singularities within the residually 

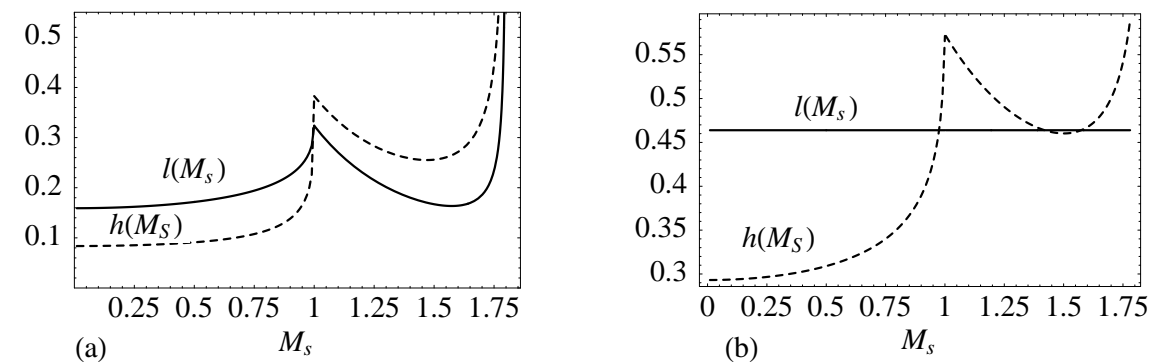

Figure 8. The dependence of the size of the plasticity region on the Mach number $M_{s}=U / c_{s}$. The figure (a) is for two dimensions and the figure (b) is for axial symmetry. For axisymmetrical geometry $l\left(M_{s}\right)$ appears to be constant.

stressed region. However, instead of using the point force singular solution, it is now appropriate to use the force dipoles or 'nuclei of strain' introduced in Love (1944) to represent the traction exerted on the boundary between the residually stressed and pristine regions. For simplicity, these nuclei of strain will be distributed along the $x$-axis in $x<U t$ or along the $z$-axis in $z<U t$. Like the point force, the nuclei of strain move with the crater velocity and they can also be conveniently represented in the form of Love stress functions. The results are easily related to the corresponding six cases mentioned above as discussed in the appendix.

\section{(iii) Total elastic response}

\section{Subsonic penetration}

For subsonic penetration, we can now model the total elastic response in the pristine region as being that due to a point force at $\xi=0$ on the axis together with an appropriate distribution of nuclei of strain along the axis, with $\xi<0$. The corresponding displacements, strain and stress components are the sum of those obtained via the Love stress functions from the boxes for $L_{F}$ in the appendix, and distributions of nuclei of strain defined as combinations of force dipoles.

The strengths of the singular solutions are calculated iteratively, the first step being to take the density $q$ of the nucleus of strain distribution so as to make the traction vanish on a cavity whose geometry we take from typical experimental measurements, i.e. we ignore the effect of the residual stress region at the first step. For example, for axisymmetric quasistatic penetration with $M_{s}=0$, we see that if we choose $L_{F}$ from (A10) with $\beta_{i} \rightarrow 1$, the traction on the cavity is

$$
\begin{aligned}
\sigma_{r r}= & -\frac{F}{4 \pi} \frac{1}{2(1-\nu)}\left(2(1+\nu) r^{2}-(1-2 \nu) \xi^{2}\right) \frac{\xi}{\left(r^{2}+\xi^{2}\right)^{5 / 2}} \\
& +\int_{-\infty}^{0} q(\eta) \frac{(\xi-\eta)^{2}-2 r^{2}}{\left(r^{2}+(\xi-\eta)^{2}\right)^{5 / 2}} d \eta .
\end{aligned}
$$

As $r \rightarrow 0$, the integral tends to

$$
\frac{q(\xi)}{r^{2}} \int_{-\infty}^{\infty} \frac{\eta^{2}-2}{\left(1+\eta^{2}\right)^{5 / 2}} d \eta=-2 \frac{q(\xi)}{r^{2}}
$$

Article submitted to Royal Society 

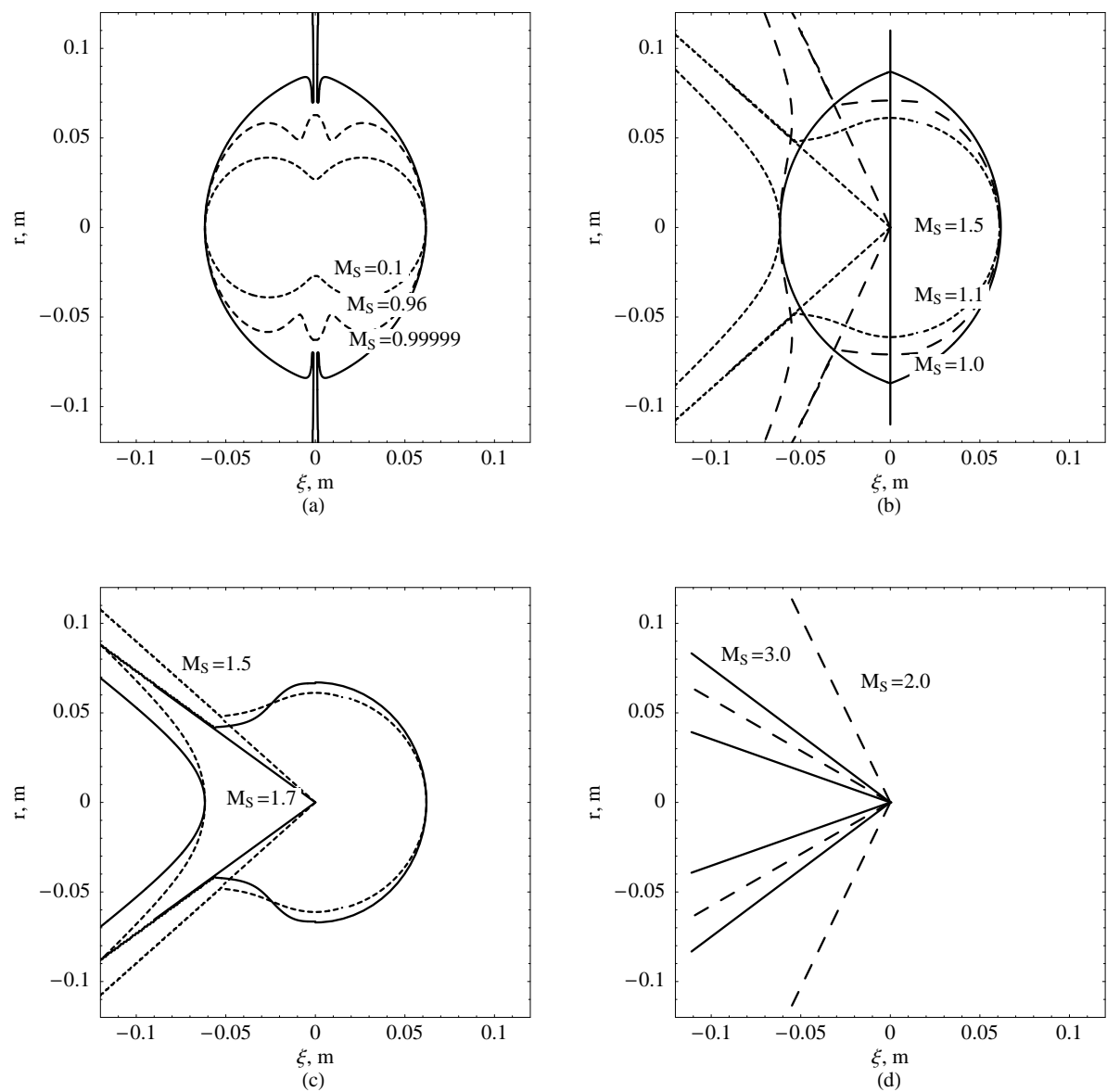

Figure 9. Dependence of the Von Mises criterion level contours $\sigma_{V M}=\sigma_{Y}$ on the Mach number $M_{s}=U / c_{s}$ for axial penetration. Curves (a) correspond to subsonic penetration with $0<M_{s}<1$, (b) to transonic penetration with $1<M_{s}<1.5$, (c) to transonic penetration with $1.5<M_{s}<c_{p} / c_{s}$ and (d) to supersonic penetration with $c_{p} / c_{s}<M_{s}$.

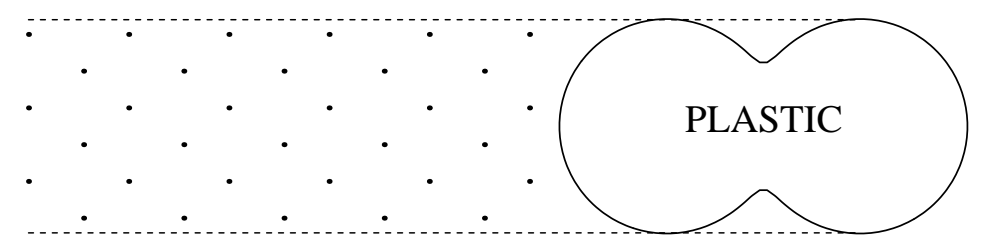

Figure 10. Schematic indication of the region of residual stress resulting from a point force.

for $\xi<0$. Hence, for a cavity of radius $a$, we choose

$$
q(\xi)=\frac{F}{16 \pi} \frac{1-2 \nu}{(1-\nu)} \frac{a^{2}}{\xi^{2}}
$$


away from the crater region. Since (3.4) is invalid when $\xi=O(r)$ and since (3.5) would lead to a divergent integral in (3.3), we truncate the integral in (3.3) to the range $-\infty<\eta<a$. This results in the Von Mises yield contour in figure $11 \mathrm{~b}$, which shows a marked contrast to that in figure 6 .

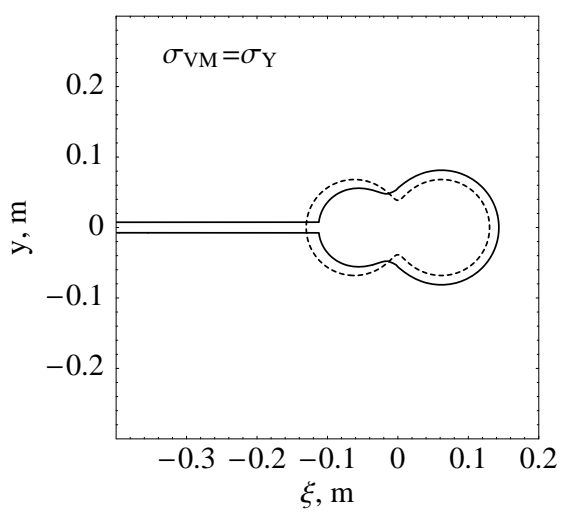

(a)

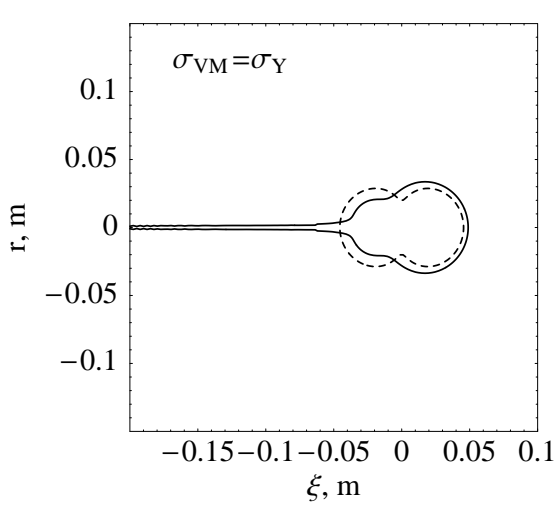

(b)

Figure 11. The Von Mises criterion contours with no traction on the cavity in comparison with the relevant contours from figures 6 , shown dashed. The figure (a) is for two dimensions and the figure (b) is for axial symmetry calculated using a discrete distribution of nuclei of strain.

The situation is quite different in two dimensions when (3.3) is replaced by

$$
\sigma_{y y}=-\frac{F \xi\left(\xi^{2} \mu-y^{2}(2 \lambda+\mu)\right)}{2 \pi\left(\xi^{2}+y^{2}\right)^{2}(\lambda+2 \mu)}+\int_{-\infty}^{0} q(\eta) \frac{(\xi-\eta)^{2}-y^{2}}{\left((\xi-\eta)^{2}+y^{2}\right)^{2}} d \eta
$$

Now the second term is a hypersingular integral that depends on the value of $q$ all along the cavity. To find the formula corresponding to (3.5) analytically we would have to solve this integral equation; we will not do this here but simply model the cavity by placing a discrete distribution of $2 n$ nuclei of strain at points $\xi_{j}$ along the negative $\xi$-axis and choosing their strengths in such a way that two components of the traction at the cavity boundary are zero at the cross sections $\xi=\xi_{j}, 1 \leq j \leq n$. The results of this are shown in figure 11a when $n=200$ and the $\xi_{j}$ are separated by 0.3 cavity radii. We note that $q$ is now of $O(F a / \xi)$.

We will now use these results as the first step in an iterative procedure that leads to an estimate of the geometry of both the plastic and residual stress regions. figure 7 suggests that the residual stress region will be cylindrical downstream of the plastic region in subsonic penetration and that it will be wedge-shaped or conical in supersonic penetration; for transonic penetration there is possibility that it will also be wedge- or cone- shaped downstream of the plastic region. In all cases we will proceed by assuming that the 'upstream' part of the elastic/plastic boundary transmits some fraction $\lambda F$ of the jet force $F$ to the pristine elastic region and that, in a frame moving with the crater, the region downstream of the plastic region comprises elastic material with a residual stress equal to that with which it 
was ejected from the plastic region; in $\S 4$ we will give an argument that suggests that $\lambda$ should be about $1 / 2$ in many circumstances. Thus, in order to improve our estimate for the elastic stresses far from the damaged region, we have to repeat the calculations that led to figure 11, but with the nucleus of strain density being chosen so that the 'residual traction' is exerted on the pristine region. For example, for quasistatic axisymmetric penetration, the calculation analogous to that leading to (3.5) shows that $q$ is related to this constant residual traction by

$$
q=\frac{F}{16 \pi} \frac{1-2 \nu}{(1-\nu)} \frac{a^{* 2}}{\xi^{2}}-\frac{1}{2} \sigma_{r r}^{*} a^{* 2},
$$

where $\sigma_{r r}^{*}$ is the residual stress at the $r=a^{*}$, the traction $\sigma_{r z}^{*}$ being an order of magnitude smaller than $\sigma_{r r}^{*}$ for small enough $a^{*}$; at the first iteration, $a^{*}=$ $h\left(M_{s}\right)\left(F / \sigma_{Y}\right)^{1 / 2}$. Having carried out this improvement, we can iterate it and, for the situation considered in figure 10, this calculation appears to converge to the configurations shown in figure 12 after around 30 iterations.

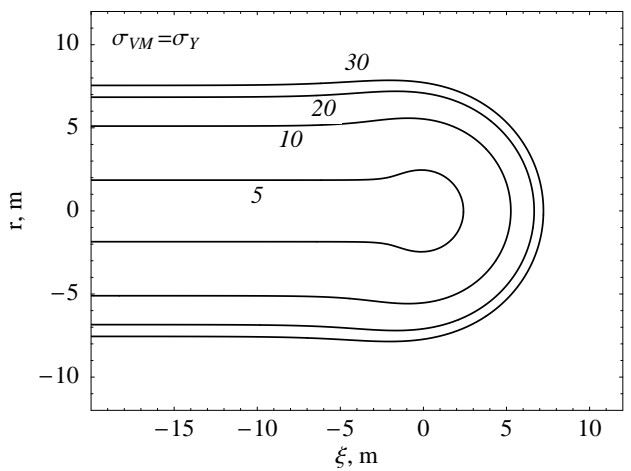

Figure 12. Residual stress region for the situation in figure 10 after 5, 10, 20 and 30 iterations.

Even in this relatively simple configuration, we must emphasize that figure 12 only gives a rough idea of the extent of the plastic region. An accurate calculation would entail solving the equations of plastic flow, which would involve computing a stress and associated flow field in the plastic region and coupling this computation to those in the elastic region via suitable mechanical and kinematic conditions at the three-component free boundary of figure 3. This coupling is associated with the fact that the plastic region is acted upon by a system of unknown sources of mass and momentum around its periphery. Moreover, even this formidable calculation should be coupled with a further free boundary model for the interaction between the jet and the crater and cavity.

The procedure that led to figure 12 can easily be repeated for the case of subsonic penetration and, for the situations depicted in figures 7, 9, the results are as shown in figure A1. These results give a clear indication that, at least for subsonic penetration, the extent of the plasticized region greatly exceeds the estimate that would result from a calculation that neglected residual stress. This is largely because of the 'spikes' in figure 7, which are the harbingers of the shock waves that appear when $U>c_{s}$. These spikes generate large regions of residual stresses, with correspondingly greater stress fields in the upstream elastic region. 


\section{Transonic and supersonic penetration}

In the transonic regime the presence of shock waves will cause major errors in our predictions wherenever it is strong enough to plasticize the target and hence ultimately to give rise to a region of residual stress. However, when the shock wave is weak, it will not affect our estimations to lowest order. Unfortunately, the position and strength of the shock can only be ascertained by solving the free boundary problem described in the previous paragraph, with the shock wave being yet another component of the 'free boundary'. All we can say for certain is that the shock wave will not originate on the axis in the pristine region because the target will plasticize there while the crater still has a small velocity compared to $c_{s}$. The shock is most likely to originate in the plastic region in the vicinity of the crater, in which case it will have decayed before it emerges into the pristine elastic region, perhaps in the configuration sketched in figure $7 \mathrm{c}$. This decay will be enhanced in the axisymmetric case because of geometrical spreading. We therefore proceed by ignoring the shock wave and iterating our stress calculation as in the subsonic case to give the results shown in figure A1b-c, e-f. Since this predicts that the presence of the residual stress region causes the plastic region to be even larger than it did in the subsonic case, we have some a posteriori justification for ignoring the shock wave.

There is, however, no possibility of ignoring shock waves in the supersonic case where $U>c_{p}$. Then there will be one or two strong shock waves which intersect the axis at a 'stand-off' distance ahead of the crater which will be of the order of the crater radius, and very rapid plasticization will occur behind the leading shock wave. Moreover, the position and strength of the shock wave(s) will now dictate the extent of the residual stress region downstream. This poses a complicated problem in the theory of solid mechanics, as discussed in Bland (1969), Germain \& Lee (1973), Thomas (1961) and we will only refer to it again briefly in the next section.

For much of the remainder of the paper we will focus on 'violent penetration', by which we mean the regime in which the plasticized region is large in all dimensions by comparison with the crater dimensions. The calculation leading to figures 7,9 and figure A1 reveals that this will only be the case when

- the jet force is such that the associated stress in the target greatly exceeds $\sigma_{Y}$ and

- we are in the subsonic or transonic regimes.

\section{Prediction of the penetration velocity}

We will now focus on the situation when the jet pressure is such that $P=\rho_{j}(V-$ $U)^{2} / 2 \gg \sigma_{Y}$, which, for subsonic and transonic penetration, is equivalent to assuming that the extent of the plastic region is $\left(P / \sigma_{Y}\right)^{\alpha} a$, where $\alpha=1$ for twodimensions and $\alpha=1 / 2$ for axial symmetry. Now the momentum equation in the plasticity region is, in Lagrangian variables,

$$
\nabla \cdot\left(\sigma^{\prime}-p \mathbf{I}\right)=\rho_{j} \frac{\partial \mathbf{v}}{\partial t},
$$

where $\mathbf{v}$ is the velocity vector, $p$ is the pressure and $\sigma^{\prime}$ is the deviatoric part of the stress tensor $\sigma$ and, in this regime, the traction applied at the crater is such that 
$p \gg\left|\sigma^{\prime}\right|$. Hence, over a large part of the plastic region we can neglect the first term in (4.1) and model the plastic flow as that of an inviscid liquid. Also, as is usual, we assume incompressibility, so that $\nabla \cdot \mathbf{v}=0$.

The violent penetration regime is the only one in which the confining elastic stresses are relatively small compared to the flow stress and hence it is the only one in which the penetration will be efficient.

One advantage of working in this regime is that we can be fairly precise about the conditions under which our 'travelling wave' assumption is justified. The theory of inviscid incompressible flow asserts that even if the jet velocity $V$ is time-dependent, the plastic flow will be quasistatic unless $V$ changes on a timescale comparable to the travel time of a target particle through the plastic region. Of course we also assume that the whole plastic region is far from the boundary of the target. Once we are working in the moving frame, the flow is steady and the plastic region in the subsonic and transonic regimes of $\S 3$ is as shown schematically in figure 13, and hence the material in this region must be slightly compressible if a travelling wave is to exist. If the relative sizes of $a$ and the plastic region were comparable, i.e. if the jet pressure $\rho_{j}(V-U)^{2}$ was comparable to $\sigma_{Y}$, then not only would the inviscid model be inappropriate but there would be no possibility that the travelling wave could exist if plastic compressibility was neglected. Indeed, since the relative volume change caused by the pressure at the cavity is of the order of $\left(P / \sigma_{Y}\right)^{1 / 2} a \times a^{2}$ divided by $\left(P / \sigma_{Y}\right)^{3 / 2} a^{3}$ in the axisymmetric case, the compressibility must be of the order of $\sigma_{Y} / P$.
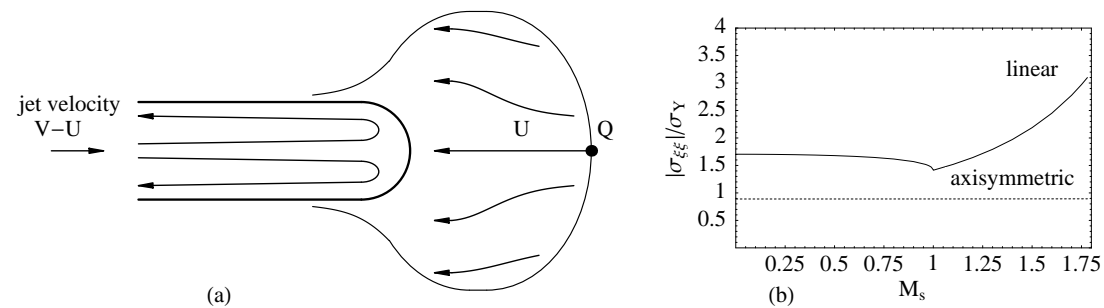

Figure 13. (a) plastic flow around the crater in a frame moving with the crater, (b) normal stress at the point $\mathrm{Q}$, which is fixed in the moving frame, as a function of the Mach number.

Far and away the most important consequence of working in the violent penetration regime is that, as anticipated in Birkhoff et al. (1948), in the subsonic and transonic regimes we do not even have to solve the force balance for the problem of figure $13 \mathrm{a}$ in order to relate $U$ to $V$. We simply use Bernoulli's equation along the stagnation streamline to say that

$$
\frac{1}{2} \rho_{j}(V-U)^{2}+p_{j}=\frac{1}{2} \rho_{t} U^{2}+p_{Q}
$$

where $p_{Q}$ is the pressure on the axis at the upstream elastic/plastic boundary. This result is independent of the geometry of figure 13a, but our earlier remark implies that it would not apply if $V$ were to change over times comparable to the travel time through the plastic region. Even more important, our assumption that $\rho_{j}(V-U)^{2} / 2 \gg \sigma_{Y}$ makes it a good approximation to neglect $p_{Q}$ in $(4.2)$ and hence retrieve (2.2). This result means that, since the penetration velocity 
must exceed at least $c_{s}$ to create a shock wave, the jet Mach number must exceed $V /\left\{c_{s}\left(1+\sqrt{\rho_{t} / \rho_{j}}\right)\right\}$ if the penetration is to be supersonic.

There are some ways by which the robust result (2.2) might be improved. One possibility is to retain the term $p_{Q}$ in (4.2), which we can do by using the modelling in $\S 3$. At the end of the iteration procedure, the normal stress in the pristine region immediately ahead of the Von Mises stress contour is $\sigma_{\xi \xi}$. These stresses are displayed in figure $13 \mathrm{~b}$. Although the normal stress is not precisely equal to $p_{Q}$, figure $13 \mathrm{~b}$ suggests that for two dimensions $p_{Q}$ becomes increasingly significant at larger Mach numbers in the transonic regime. The effect is not present in axisymmetric penetration because of the constancy of $l\left(M_{s}\right)$ in figure $8 \mathrm{~b}$.

Alternatively, we can consider the possible implication of our 'inviscid plasticity' model for unsteady penetration, still under the assumption that $\rho_{j}(V-U)^{2} / 2 \gg$ $\sigma_{Y}$, so that the plasticity region is large compared to the cavity radius but without the timescale constraint mentioned above. For such unsteady flows, Bernoulli's equation on the centre line now depends on the global properties of the plastic flow. Hence we must consider the response of the whole plastic region not just to the jet pressure $p_{j}$ at the crater but to the total force $F$ exerted by the jet on the crater. It is possible to take the analysis further if we assume the plastic flow is irrotational, so that $\mathbf{v}=\nabla \phi$, where the velocity potential $\phi$ is related to $p$ by

$$
\frac{p}{\rho_{t}}+\frac{\partial \phi}{\partial t}+\frac{1}{2}|\nabla \phi|^{2}=0
$$

assuming $p$ and $\mathbf{v}$ are negligible far from the crater. Also, since the crater is small in comparison with the heavily plasticized region, the local flow in the vicinity of the crater is that of quasistatic penetration of an inviscid liquid by a cavity moving with velocity $U$. Now let us recall some results from the theory of such quasistatic cavity flows, be they two dimensional (Birkhoff \& Zarantonello 1957; Milne-Thomson 1968) or axisymmetric (Birkhoff \& Zarantonello 1957; Levinson 1946). In two dimensions, the velocity field is proportional to $U$ and decays like the inverse square root of distance from the crater. With axial symmetry the decay rate has an extra logarithmic factor associated with the Legendre function $R^{1 / 2} P_{1 / 2}(\cos \theta)$ in spherical polar coordinates $(R, \theta)$ (Levinson 1946). These decay rates govern the force exerted on the target by the penetrating cavity because they enable us to make a simple calculation of the pressures acting on a control volume surrounding the crater. Such a calculation shows that the force on the 'upstream' free boundary in figure 3 is $F / 2$, which suggests that we use the value $\lambda=1 / 2$ in the iteration procedure in $\S 3 a$ (iii). In either case, away from the crater, $\phi$ will be a function of $\left(x-\int_{0}^{t} U(\tau) d \tau, y\right)$ or $\left(z-\int_{0}^{t} U(\tau) d \tau, r\right)$ multiplied by $U$ and (4.3) will predict that the pressure in the plastic region satisfies

$$
\frac{p}{\rho_{t}}+\text { const. } \frac{d U}{d t}=\frac{1}{2} U^{2}+\frac{p_{Q}}{p_{t}}
$$

the constant being determined by matching the local flow to the far field. This constant is associated with the inertia of the plastic region and is proportional to the time taken to attain the travelling wave starting from rest, and (4.4) means that (4.2) will now become a differential equation for $U$. 
Finally let us briefly return to the regime of supersonic penetration. In this regime, the scenario analogous to that of figure 13a requires the pristine material to pass through some combination of shock waves and plasticity waves before it flows plastically around the crater. We can still use Bernoulli's equation in cases of violent penetration, but now (4.2) becomes

$$
\frac{1}{2} \rho_{j}(V-U)^{2}+p_{j}=\frac{1}{2} \rho_{t} \tilde{U}^{2}+\tilde{p},
$$

where $\tilde{U}$ and $\tilde{p}$ are the velocity and pressure with which the target material on the axis emerges from the shock/plasticity wave. For sufficiently large $V, U-\tilde{U}$ and $\tilde{p}$ will no longer be negligible compared to $U, p_{j}$ respectively and (2.2) will no longer apply. This is an interesting area for future research, and here we simply point out that there is a possible analogy with the theory of incompressible hypersonic blunt body flows in gasdynamics (Hayes \& Probstein 1959).

\section{Conclusions}

This paper has described a scenario within which to model shaped charge penetration systematically. The model that emerges is that of a multiple free boundary problem in which the free boundaries connect region of the target that are pristine elastic, residually stressed elastic and plastic respectively.

Although this free boundary problem can only be solved numerically, we have obtained estimates for the sizes of the different regions by carrying out an iteration starting from the purely elastic response. This iteration strongly relies on the use of appropriate Love stress functions which depend on whether or not the penetration is subsonic, transonic or supersonic. The resulting stress fields show that effective penetration is only achieved when the pressure in the crater is much greater than the yield stress in the target.

In this particular parameter regime, a considerable part of the plastic flow region can be modelled as an inviscid liquid, enabling the classic result of Birkhoff et al. (1948) to be retrieved. Our scenario suggests ways in which this result would be modified to account for target confinement and unsteady effects. Both these modifications lead to interesting open mathematical problems even for subsonic and transonic penetration, while the case of supersonic penetration still presents a fundamental challenge.

We remark that shaped charge penetration offers an interesting contrast to other penetration mechanisms in continuum mechanics. Of these, the best studied is that of penetration of an inviscid liquid by a rigid body. In this case, it is the inertia of the penetrating body that controls the dynamics, with the drag force exerted by the cavitating flow balancing the rate of change of momentum of the body. The same idea applies to the other extreme in which non-fracturing elastic solid is penetrated by a rigid body, when the resisting force is an elastic contact force. In such cases the penetration will be shallow unless the target is rubber-like. It may therefore seem strange that in shaped charge penetration, the penetration velocity can be estimated without having to calculate any resisting force, at least in the travelling wave scenario of $\S 4$. However, there has been an underlying assumption about the resisting force exerted by the target, namely that it is associated with a stress field much greater than the yield stress. Also we have made this assumption 
based on the experimentally-observed cavity thickness. This has led us to a model analogous to that of inviscid target penetration by a solid projectile, but with the penetrator being a jet of prescribed velocity, and this has made the travelling wave calculation leading to (4.2) very easy. Were the jet pressure to be insufficient for this assumption, we would be forced to carry out a much more complicated analysis near the crater in which the cavity thickness would be unknown, and in which the resisting force would appear explicitly.

Finally, we note that we have not needed to use any energy balance argument, such as would have been necessary had there been significant fracture or melting of the target. Indeed, theoretical scenario could, in principle, be used to predict thermal dissipation via an uncoupled calculation. This situation has came about because the violent penetration assumption is based purely on momentum considerations; for less violent penetration, the energy balance considered in Walker (2001) becomes increasingly important.

The authors thank Dr J. Curtis, Dr C. Poole, Dr D.J. Allwright, Prof. S. Reid, Dr. N. Petrinic, Prof. N. Bourne, Dr G. Kozyreff for helpful comments and suggestions, and QinetiQ, EPSRC and Smith Institute for financial support and technology transfer.

\section{References}

Backman, M.E. \& Goldsmith, W. 1978 The mechanics of penetration of projectiles into targets. Int. J. Engng. Sci., 16, 1-99.

Barnea, N. \& Sela, N. 1996 Why do shaped charge jets cease penetrating? In Proc. 16th Int. Symposium of Ballistics, San Francisco, 1996, pp. 349-358.

Birkhoff, G., Macdougall, D.P., Pugh, E.M. \& Taylor G.I. 1948 Explosives with lined cavities. J. Appl. Phys. 19, 563-582.

Birkhoff, G. \& Zarantonello, E.H. 1957 Jets, wakes and cavities. New York: Academic Press.

Bland, D.R. 1969 Nonlinear dynamic elasticity. Blaisdell.

Christman, D.R. \& Gehring, J.W. 1966 Analysis of high-velocity projectile penetration mechanics. J. Appl. Phys. 37, 1579-1587.

Cook, M.A. 1959 Mechanism of cratering in ultra-high velocity impact. J. Appl. Phys. 30, $725-735$

Cornish, R. 2001 A 3D modelling study of the influence of slide wall collision on long standoff jet penetration. In Proc. 19th International Symposium of Ballistics, Interlaken, 2001, pp. 1545-1552.

Duan, Z.Q., Li, S.X. \& Huang, D.W. 2003 Microstructures and adiabatic shear bands formed by ballistic impact in steels and tungsten alloy. Fatigue Fract. Engn. Mater. Struct. 26, 1119-1126.

Eason, G., Fulton, J. \& Sneddon, I.N. 1956 The generation of waves in an infinite elastic solids by variable body forces. Phil. Trans. Roy. Soc. Lon. 248, 575-607.

Eddingfield, D., Evers, J.L. \& Setork, A. 1981 Mathematical modelling of high velocity water jets. In Proc. 1st US water jet conference, 1981, pp. 25-39.

Eichelberger, R.J. \& Pugh, E.M. 1952 Experimental verification of jet formation by charges with lined conical cavities. J. Appl. Phys. 23, 527-531.

Evans, W.M. \& Pack, D.C. 1951 Penetration by high-velocity ('Munroe') jets: II. Proc. Phys. Soc., 64, 303-310.

Freund, L.B. 1998 Dynamic fracture mechanics. Cambridge University Press. 
Germain, P. \& Lee, E.H. 1973 On shock waves in elastic-plastic solids. J. Mech. Phys. Solids 21, 359-382.

Hayes, W.D. \& Probstein, R.F. 1959 Hypersonic flow theory. Academic press.

Hebden, C. 1999 Penetration mechanics of a shaped charge. M.Phil. thesis, Oxford University.

Hill, R. 1950 The mathematical theory of plasticity. Oxford University Press.

Hill, R., Mott, N.F. \& Pack, D.C. 1944 Ministry of Supply Report.

Hopkins, D.F. \& Robertson, J.M. 1967 Two-dimensional incompressible fluid jet penetration. J. Fluid Mech. 29, 273-287.

Huntington-Thresher, W.K.E., Curtis, J.P., Greenwood, P.R., Moss, P. \& Smethurst, J. 2001 Assessment of shaped charge jet mitigation, and the development of a hydrocode, analytical model link. In Proc. 19th International Symposium of Ballistics, Interlaken, 2001, pp. 897-904.

Levinson, N. 1946 On the asymptotic shape of the cavity behind an axially symmetric nose moving through an ideal fluid. Ann.of Math. 47, 704-730.

Love, A.E.H. 1944 A treatise on the mathematical theory of elasticity. New York: Dover publications.

Milne-Thomson, L.M. 1968 Theoretical hydrodynamics. 5th edn. Dover publications.

Murr, L.E., Niou, C.S., Garcia, E.P., Ferreyra, E., Rivas, J.M. \& Sanchez, J.C. 1997 Comparison of jetting-related microstructures associated with hypervelocity impact crater formation in copper targets and copper shaped charges. Mat. Sc. Eng. A 222, 118-132.

Murr, L.E., Ferreyra, E., Pappu, S., Garcia, E.P., Sanchez, J.C., Huang, W., Rivas, J.M., Kennedy, C., Ayala, A. \& Niou, C.S. 1996 Novel deformation processes and microstructures involving ballistic penetrator formation and hypervelocity impact and penetration phenomena. Mat. Charact. 37, 245-276.

Ockendon, H. \& Ockendon, J.R. 2004 Waves and compressible flow. Springer.

Ockendon, J.R., Howison, S., Lacey, A. \& Movchan, A. 2003 Applied partial differential equations. Oxford University Press.

Pack, D.C. \& Evans, W.M. 1951 Penetration by high-velocity ('Munroe') jets: I. Proc. Phys. Soc. 64, 298-302.

Poole, C.J. 2005 Shaped charge mechanics. D.Phil. thesis, Oxford University.

Radok, J.R.M. 1956 On the solution of problems of dynamic plane elasticity. Quarterly of Applied Mathematics 14, 289-298.

Ravid, M., Hirsch, E., Shinar, G.I. \& Barnea, N. 1995 An analytical model for the cratering of metallic targets by hypervelocity long rods. In Proc. 15th Int. Symposium of Ballistics, Jerusalem, 1995, pp. 211-218.

Sneddon, I.N. \& Berry, D.S. 1958 The classical theory of elasticity. New York: SpringerVerlag.

Swanson, R.K., Kilman, M., Cerwin, S. \& Tarver, W. 1987 Study of particle velocities in water driven abrasive jet cutting. In Proc. 4th US water jet conference, 198\%, pp. 163171.

Szendrei, T. 1995 Analytical model for high-velocity impact cratering with material strength: extensions and validation. In Proc. 15th Int. Symposium of Ballistics, Jerusalem, 1995, pp. 123-131.

Szendrei, T. 1998 Link between axial penetration and radial crater expansion in hypervelocity impact. In Proc. 17th Int. Symposium of Ballistics, Midrand, 1998, pp. 325-332.

Tate, A. 1986 a Long rod penetration models, part i: a flow field model for high speed long rod penetration. Int. J. Mech. Sci., 28, 535-548.

Tate, A. $1986 b$ Long rod penetration models, part ii: extentions to the hydrodynamic theory of penetration. Int. J. Mech. Sci., 28, 599-612. 
Thomas, T.Y. 1961 Plastic flow and fracture in solids. Academic press.

Walker, J.D. 2001 Hypervelocity penetration modeling: momentum vs. energy and energy transfer mechanisms. Int. J. Imp. Eng., 26, 809-822.

Walker, J.D. \& Anderson, C.E. 1995 A time-dependent model for long-rod penetration. Int. J. Imp. Eng., 16, 19-48.

Walters, W.P. \& Zukas, J.A. 1989 Fundamentals of shaped charges. John Wiley \& Sons.

Watkins, R. 2000 Asymptotics of shaped charge jet penetration. M.Phil. thesis, Oxford University.

Watson, A.J. \& Moxon, C.J. 1985 Ultra high velocity water jet. In Proc. 3rd US water jet conference, 1985, pp. 117-131.

Wells, J.M., Green, W.H., Rupert, N.L., Cole, A., Alkemade, S.J., Cimpoeru, S.J. \& Barnea, N. 2002 Ballistic damage visualization in monolithic Ti-6Al-4V with X-ray computed tomography., In Proc. 20th Int. Symposium of Ballistics, Orlando, 2002, pp. 1112-1120.

Yin, Z.X., Ma, C.M., Li, S.X. \& Cheng, G.D. 2004 Perforation of an ultra-high strength steel penetrated by shaped charge jet. Mat. Sc. Eng. A 379, 809-822.

\section{Appendix A.}

\section{(a) Dynamic Love stress functions}

By analogy with the biharmonic stress function for axisymmetric elastostatics introduced in Love (1944), we can use dynamic stress functions $L(x, y, t)$ in plane strain (with loading only in, say, the $x$-direction) and $L(z, r, t)$ in axial symmetry. These functions are related to the displacements $\left(u_{x}, u_{y}\right)$ and $\left(u_{z}, u_{r}\right)$ by:

$$
\begin{gathered}
u_{x}=\frac{1}{2 \mu} \frac{1}{1-\nu}\left((1-2 \nu)\left(\nabla^{2}-\frac{1}{c_{s}^{2}} \frac{\partial^{2}}{\partial t^{2}}\right)+\frac{\partial^{2}}{\partial y^{2}}\right) L, \quad u_{y}=-\frac{1}{2 \mu} \frac{1}{1-\nu} \frac{\partial^{2} L}{\partial x \partial y} \\
\sigma_{x x}=\frac{1}{1-\nu} \frac{\partial}{\partial x}\left((1-\nu)\left(\nabla^{2}-\frac{1}{c_{s}^{2}} \frac{\partial^{2}}{\partial t^{2}}\right)+\frac{\partial^{2}}{\partial y^{2}}\right) L, \quad \nabla^{2}=\frac{\partial^{2}}{\partial x^{2}}+\frac{\partial^{2}}{\partial y^{2}} \\
\sigma_{x y}=\frac{1}{1-\nu} \frac{\partial}{\partial y}\left(-\nu \nabla^{2}-\frac{1}{2}(1-2 \nu) \frac{1}{c_{s}^{2}} \frac{\partial^{2}}{\partial t^{2}}+\frac{\partial^{2}}{\partial y^{2}}\right) L \\
\sigma_{y y}=\frac{1}{1-\nu} \frac{\partial}{\partial x}\left(\nu\left(\nabla^{2}-\frac{1}{c_{s}^{2}} \frac{\partial^{2}}{\partial t^{2}}\right)-\frac{\partial^{2}}{\partial y^{2}}\right) L
\end{gathered}
$$

and

$$
\begin{gathered}
u_{z}=\frac{1}{2 \mu} \frac{1}{1-\nu}\left((1-2 \nu)\left(\nabla^{2}-\frac{1}{c_{s}^{2}} \frac{\partial^{2}}{\partial t^{2}}\right)+\frac{\partial^{2}}{\partial r^{2}}+\frac{1}{r} \frac{\partial}{\partial r}\right) L, u_{r}=-\frac{1}{2 \mu} \frac{1}{1-\nu} \frac{\partial^{2} L}{\partial r \partial z}, \\
\sigma_{r r}=\frac{1}{1-\nu} \frac{\partial}{\partial z}\left(\nu\left(\nabla^{2}-\frac{1}{c_{s}^{2}} \frac{\partial^{2}}{\partial t^{2}}\right)-\frac{\partial^{2}}{\partial r^{2}}\right) L, \quad \nabla^{2}=\frac{\partial^{2}}{\partial z^{2}}+\frac{1}{r} \frac{\partial}{\partial r}+\frac{\partial^{2}}{\partial r^{2}}, \\
\sigma_{z z}=\frac{1}{1-\nu} \frac{\partial}{\partial z}\left((1-\nu)\left(\nabla^{2}-\frac{1}{c_{s}^{2}} \frac{\partial^{2}}{\partial t^{2}}\right)+\frac{1}{r} \frac{\partial}{\partial r}+\frac{\partial^{2}}{\partial r^{2}}\right) L, \\
\sigma_{\theta \theta}=\frac{1}{1-\nu} \frac{\partial}{\partial z}\left(\nu\left(\nabla^{2}-\frac{1}{c_{s}^{2}} \frac{\partial^{2}}{\partial t^{2}}\right)-\frac{1}{r} \frac{\partial}{\partial r}\right) L, \\
\sigma_{r z}=\frac{1}{1-\nu} \frac{\partial}{\partial r}\left((1-\nu) \nabla^{2}-\frac{1}{2}(1-2 \nu) \frac{1}{c_{s}^{2}} \frac{\partial^{2}}{\partial t^{2}}-\frac{\partial^{2}}{\partial z^{2}}\right) L .
\end{gathered}
$$


Table 2.

\begin{tabular}{lll}
\hline & Two-dimensions $(\xi, y)$ & Axial symmetry $(\xi, r)$ \\
\hline$\nabla_{s}^{2}$ & $\left(\beta_{s}^{2} \frac{\partial^{2}}{\partial \xi^{2}}+\frac{\partial^{2}}{\partial y^{2}}\right)$ & $\left(\beta_{s}^{2} \frac{\partial^{2}}{\partial \xi^{2}}+\frac{1}{r} \frac{\partial}{\partial r}+\frac{\partial^{2}}{\partial r^{2}}\right)$ \\
$\nabla_{p}^{2}$ & $\left(\beta_{p}^{2} \frac{\partial^{2}}{\partial \xi^{2}}+\frac{\partial^{2}}{\partial y^{2}}\right)$ & $\left(\beta_{p}^{2} \frac{\partial^{2}}{\partial \xi^{2}}+\frac{1}{r} \frac{\partial}{\partial r}+\frac{\partial^{2}}{\partial r^{2}}\right)$ \\
$\square_{s}^{2}$ & $\left(-B_{s}^{2} \frac{\partial^{2}}{\partial \xi^{2}}+\frac{\partial^{2}}{\partial y^{2}}\right)$ & $\left(-B_{s}^{2} \frac{\partial^{2}}{\partial \xi^{2}}+\frac{1}{r} \frac{\partial}{\partial r}+\frac{\partial^{2}}{\partial r^{2}}\right)$ \\
$\square_{p}^{2}$ & $\left(-B_{p}^{2} \frac{\partial^{2}}{\partial \xi^{2}}+\frac{\partial^{2}}{\partial y^{2}}\right)$ & $\left(-B_{p}^{2} \frac{\partial^{2}}{\partial \xi^{2}}+\frac{1}{r} \frac{\partial}{\partial r}+\frac{\partial^{2}}{\partial r^{2}}\right)$
\end{tabular}

We remark that in static elasticity these definitions can easily be obtained from the fundamental Galerkin solution $2 \mu(1-\nu) \mathbf{u}=2(1-\nu) \nabla^{2} \mathbf{B}-\nabla(\nabla \cdot \mathbf{B})$ of the Navier equation, where $\nabla^{4} \mathbf{B}=\mathbf{0}$, with the assumption that, in two-dimensions, $\mathbf{B}=L(x, y) \mathbf{e}_{x}, \nabla^{4} L=0$ or, with axial symmetry, $\mathbf{B}=L(z, r) \mathbf{e}_{z}, \nabla^{4} L=0$. In elastodynamics, the corresponding Love stress functions defined by (A 1)-(A 2) satisfy

$$
\left(\nabla^{2}-\frac{1}{c_{s}^{2}} \frac{\partial^{2}}{\partial t^{2}}\right)\left(\nabla^{2}-\frac{1}{c_{p}^{2}} \frac{\partial^{2}}{\partial t^{2}}\right) L=0 .
$$

Travelling wave solutions $\widetilde{L}(x-U t, y)$ or $\widetilde{L}(z-U t, r)$ satisfy the equations.

$$
\nabla_{s}^{2} \nabla_{p}^{2} \widetilde{L}=0, \quad \square_{s}^{2} \nabla_{p}^{2} \widetilde{L}=0, \quad \square_{s}^{2} \square_{p}^{2} \widetilde{L}=0
$$

in the subsonic, transonic and supersonic regimes respectively, where $\xi=x-U t$ or $\xi=z-U t$ and the operators are depicted in table 2. The equations in twodimensions were independently obtained by Radok (1956) and Sneddon \& Berry (1958). Here $\beta_{i}^{2}=1-M_{i}^{2}, B_{i}^{2}=M_{i}^{2}-1$ and $M_{i}=U / c_{i}$ are the Mach numbers, and $c_{p}=\sqrt{(\lambda+2 \mu) / \rho}, c_{s}=\sqrt{\mu / \rho}$ are the sound propagation velocities.

\section{(i) Point forces}

A point force $F$ applied in the $\xi$-direction at the origin is modelled by a term $F \delta(\xi) \delta(y)$ or $F \delta(x) \delta(y) \delta(\xi)$ on the right-hand side of the equations (A 3). These equations then have to be solved subject to the condition that the stress at infinity is equivalent to an equal and opposite force.

\section{Subsonic penetration}

In two-dimensions we have to solve the equation

$$
\nabla_{s}^{2} \nabla_{p}^{2} \widetilde{L}(\xi, y)=F \delta(\xi) \delta(y)
$$

so that

$$
\nabla_{p}^{2} \widetilde{L}(\xi, y)=\frac{F}{2 \pi} \frac{1}{\beta_{s}} \ln \sqrt{\frac{\xi^{2}}{\beta_{s}^{2}}+y^{2}} \quad \text { with } \widetilde{L}=o\left(\left(\xi^{2}+y^{2}\right)^{3 / 2}\right) \text { at } \infty
$$


In the complex variables $z=\xi / \beta_{s}+\mathrm{i} y, \bar{z}=\xi / \beta_{s}-\mathrm{i} y$ and $\lambda=\beta_{p} / \beta_{s}$

$$
\left(\left(\lambda^{2}-1\right)\left(\frac{\partial^{2}}{\partial z^{2}}+\frac{\partial^{2}}{\partial \bar{z}^{2}}\right)+2\left(\lambda^{2}+1\right) \frac{\partial^{2}}{\partial z \partial \bar{z}}\right) \widetilde{L}=\frac{F}{4 \pi \beta_{s}}(\ln z+\ln \bar{z}),
$$

with a particular solution

$$
\widetilde{L}=\frac{F}{4 \pi \beta_{s}} \frac{1}{\lambda^{2}-1}\left(\frac{1}{2}\left(z^{2} \ln z+\bar{z}^{2} \ln \bar{z}\right)-\frac{3}{4}\left(z^{2}+\bar{z}^{2}\right)\right) .
$$

Hence, with $X=\xi / \beta_{s}$,

$$
\begin{aligned}
\widetilde{L}(X, y)= & \frac{F}{2 \pi \beta_{s}} \frac{1}{\lambda^{2}-1}\left(\frac{1}{2}\left(\lambda^{2} X^{2}-y^{2}\right) \ln \sqrt{\lambda^{2} X^{2}+y^{2}}-\lambda X y \arctan \frac{y}{\lambda X}\right. \\
& \left.-\frac{3}{4}\left(\lambda^{2} X^{2}-y^{2}\right)\right)+\Phi(X, y),
\end{aligned}
$$

where $\Phi$ is that harmonic function which ensures that (A 5) is satisfied. Apart from terms linear in $(X, y)$

$$
\begin{aligned}
\Phi(X, y)= & -\frac{F}{2 \pi \beta_{s}} \frac{\lambda}{\lambda^{2}-1}\left(\frac{1}{2}\left(X^{2}-y^{2}\right) \ln \sqrt{X^{2}+y^{2}}-X y \arctan \frac{y}{X}\right. \\
& \left.-\frac{3}{4}\left(X^{2}-y^{2}\right)\right)
\end{aligned}
$$

and the final solution of the equation (A 4) becomes:

$$
\begin{aligned}
\widetilde{L}_{F}(\xi, y)= & -\frac{F}{8 \pi} \frac{1}{\beta_{s}^{2}-\beta_{p}^{2}}\left(\left(2 \beta_{s} d_{s}^{2} \ln r_{s}-4 \xi y \arctan \frac{\beta_{s} y}{\xi}-3 \beta_{s} d_{s}^{2}\right)\right. \\
& \left.-\left(2 \beta_{p} d_{p}^{2} \ln r_{p}-4 \xi y \arctan \frac{\beta_{p} y}{\xi}-3 \beta_{p} d_{p}^{2}\right)\right)
\end{aligned}
$$

where

$$
d_{s}^{2}=\frac{\xi^{2}}{\beta_{s}^{2}}-y^{2}, \quad d_{p}^{2}=\frac{\xi^{2}}{\beta_{p}^{2}}-y^{2}, \quad r_{s}^{2}=\frac{\xi^{2}}{\beta_{s}^{2}}+y^{2}, \quad r_{p}^{2}=\frac{\xi^{2}}{\beta_{p}^{2}}+y^{2} .
$$

With axial symmetry, we proceed similarly. Since

$$
\nabla_{s}^{2} \nabla_{p}^{2} \widetilde{L}(\xi, r)=F \delta(x) \delta(y) \delta(\xi),
$$

with zero stress at infinity, we solve the equation

$$
\nabla_{p}^{2} \widetilde{L}(\xi, r)=\frac{F}{4 \pi \beta_{s}} \frac{1}{\sqrt{\frac{\xi^{2}}{\beta_{s}^{2}}+r^{2}}}, \quad \text { with } \widetilde{L}=o\left(\left(r^{2}+\xi^{2}\right)^{1 / 2}\right) \text { at } \infty .
$$

Hence, by adding a suitable potential function to a particular solution of (A 12),

$$
\begin{aligned}
\widetilde{L}(Z, r)= & F \frac{\alpha_{2}}{\alpha_{1}}\left(\frac{1}{\lambda}\left(\sqrt{\lambda^{2} Z^{2}+r^{2}}+\frac{1}{2} \lambda Z \ln \frac{\sqrt{\lambda^{2} Z^{2}+r^{2}}-\lambda Z}{\sqrt{\lambda^{2} Z^{2}+r^{2}}+\lambda Z}\right)\right. \\
& \left.-\left(\sqrt{Z^{2}+r^{2}}+\frac{1}{2} Z \ln \frac{\sqrt{Z^{2}+r^{2}}-Z}{\sqrt{Z^{2}+r^{2}}+Z}\right)\right),
\end{aligned}
$$


where

$$
\alpha_{1}=-\frac{1}{2 \mu(1-\nu)} \frac{1}{\beta_{p}}, \quad \alpha_{2}=\frac{1}{4 \pi \mu} \frac{1}{1-\beta_{s}^{2}}, \quad \nu=\frac{1}{2}\left(1+\frac{\beta_{p}^{2}-1}{\beta_{p}^{2}-\beta_{s}^{2}}\right)
$$

The final solution of the equation (A 11) becomes

$$
\widetilde{L}_{F}(\xi, r)=-\frac{F}{8 \pi} \frac{1}{\beta_{s}^{2}-\beta_{p}^{2}}\left(2\left(\beta_{p} r_{p}-\beta_{s} r_{s}\right)+\xi \ln \frac{\left(r_{p}-\xi / \beta_{p}\right)\left(r_{s}+\xi / \beta_{s}\right)}{\left(r_{p}+\xi / \beta_{p}\right)\left(r_{s}-\xi / \beta_{s}\right)}\right),
$$

where

$$
r_{s}^{2}=\frac{\xi^{2}}{\beta_{s}^{2}}+r^{2}, \quad r_{p}^{2}=\frac{\xi^{2}}{\beta_{p}^{2}}+r^{2}
$$

\section{Transonic penetration}

In the two-dimensional case

$$
\square_{s}^{2} \nabla_{p}^{2} \widetilde{L}(\xi, y)=F \delta(\xi) \delta(y), \quad \text { with } \widetilde{L}=o\left(r^{2}+\xi^{2}\right) \text { at } \infty,
$$

and $\widetilde{L}$ satisfies the condition that there is no upstream propagation of $S$-waves into $\xi>0$. Writing $\Psi=\nabla_{p}^{2} \widetilde{L}$

$$
\square^{2} \Psi\left(\xi / B_{s}, y\right)=\frac{F}{B_{s}} \delta\left(\xi / B_{s}\right) \delta(y)
$$

where

$$
\square^{2}=-\frac{\partial^{2}}{\partial X^{2}}+\frac{\partial^{2}}{\partial y^{2}}, \quad X=\xi / B_{s} .
$$

The relevant solution of the equation (A 16) is:

$$
\Psi(X, y)=-\frac{F}{4 B_{s}} H(-(X-y)) H(-(X+y))
$$

where $H(\cdot)$ is the Heaviside function, with

$$
H(x)= \begin{cases}0, & x<0 \\ 1, & x>0\end{cases}
$$

Hence

$$
\nabla_{p}^{2} \widetilde{L}(\xi, y)=-\frac{F}{4 B_{s}} H\left(-\left(\xi / B_{s}-y\right)\right) H\left(-\left(\xi / B_{s}+y\right)\right)
$$

with

$$
\nabla^{2} \widetilde{L}(X, y)=-\frac{F}{4 B_{s}} H(-(\lambda X-y)) H(-(\lambda X+y))
$$


and the final solution of (A 15) in which $\widetilde{L}$ and $\nabla \widetilde{L}$ are continuous and $\widetilde{L}$ satisfies a minimum growth condition at infinity is

$$
\widetilde{L}(\xi, y)=B \begin{cases}B_{s} \pi r_{s}^{2}-\omega, & |y| \leq|\xi| / B_{s}, \xi<0, \\ -2 \pi y \xi-\omega, & y>-\xi / B_{s}, \quad \xi<0, \\ & \text { or } y<0, \quad \xi>0, \\ 2 \pi y \xi-\omega, & y<\xi / B_{s}, \quad \xi<0, \\ & \text { or } y>0, \quad \xi>0,\end{cases}
$$

where

$$
\begin{gathered}
\omega=2 y \xi \arctan \frac{\beta_{p} y}{\xi}+d_{p}^{2} \beta_{p} \log r_{p}, \\
B=-\frac{F}{4 \pi} \frac{\beta_{p} B_{s}}{B_{s}^{2}+\beta_{p}^{2}}, \quad r_{s}^{2}=\frac{\xi^{2}}{B_{s}^{2}}+y^{2}, \quad r_{p}^{2}=\frac{\xi^{2}}{\beta_{p}^{2}}+y^{2}, \quad d_{p}^{2}=\frac{\xi^{2}}{\beta_{p}^{2}}-y^{2} .
\end{gathered}
$$

Similarly, in the axially symmetric case, we need to solve

$$
\square_{s}^{2} \nabla_{p}^{2} \widetilde{L}(\xi, r)=F \delta(x) \delta(y) \delta(\xi)
$$

with the same condition at infinity as in (A 15). Again we write $\Psi=\nabla_{p}^{2} \widetilde{L}$ and the substitution $\xi=B_{s} Z$ leads to the equation

$$
\square^{2} \Psi(Z, r)=\frac{F}{B_{s}} \delta(x) \delta(y) \delta(Z), \quad \square^{2}=-\frac{\partial^{2}}{\partial Z^{2}}+\frac{1}{r} \frac{\partial}{\partial r}+\frac{\partial^{2}}{\partial r^{2}},
$$

whose relevant solution is

$$
\Psi(Z, r)= \begin{cases}0, & r>-Z, \\ \frac{F}{4 \pi} \frac{1}{B_{s}} \frac{1}{\sqrt{Z^{2}-r^{2}}}, & r<-Z .\end{cases}
$$

Hence we solve

$$
\nabla_{p}^{2} \widetilde{L}(\xi, r)= \begin{cases}0, & r>-\xi / B_{s}, \\ \frac{F}{4 \pi} \frac{1}{B_{s}} \frac{1}{\sqrt{\xi^{2} / B_{s}^{2}-r^{2}}}, & r<-\xi / B_{s}\end{cases}
$$

and $\widetilde{L}$ and $\nabla \widetilde{L}$ are continuous on $r=-\xi / B_{s}$. Again we use the substitution $\xi=\beta_{p} Z$ and $\lambda=\beta_{p} / B_{s}$ which give

$$
\nabla^{2} \widetilde{L}= \begin{cases}0, & r>-\lambda Z, \\ \frac{F}{4 \pi} \frac{1}{B_{s}} \frac{1}{\sqrt{\lambda^{2} Z^{2}-r^{2}}}, & r<-\lambda Z\end{cases}
$$

and the solution can be written as follows

$$
\widetilde{L}=F \frac{\alpha_{2}}{\alpha_{1}}\left\{\begin{array}{cc}
\frac{1}{\lambda} \sqrt{\lambda^{2} Z^{2}-r^{2}}-\sqrt{Z^{2}+r^{2}} & r<-\lambda Z, \\
+\frac{1}{2} Z \ln -\frac{\left(\sqrt{\lambda^{2} Z^{2}-r^{2}}-\lambda Z\right)\left(\sqrt{Z^{2}+r^{2}}+Z\right)}{\left(\sqrt{\lambda^{2} Z^{2}-r^{2}}+\lambda Z\right)\left(\sqrt{Z^{2}+r^{2}}-Z\right)}, & \\
-\left(\sqrt{Z^{2}+r^{2}}+\frac{1}{2} Z \ln \frac{\sqrt{Z^{2}+r^{2}}-Z}{\sqrt{Z^{2}+r^{2}}+Z}\right), & r>-\lambda Z,
\end{array}\right.
$$


where

$$
\alpha_{1}=-\frac{1}{2 \mu(1-\nu)} \frac{1}{\beta_{p}}, \quad \alpha_{2}=\frac{1}{4 \pi \mu} \frac{1}{1+B_{s}^{2}}, \quad \nu=\frac{1}{2}\left(1+\frac{\beta_{p}^{2}-1}{\beta_{p}^{2}+B_{s}^{2}}\right) .
$$

In $(\xi, r)$ coordinates the solution becomes:

$$
\widetilde{L}_{F}(\xi, r)=B \begin{cases}2 B_{s} d_{s}-2 \beta_{p} r_{p}+\xi \ln -\frac{\left(d_{s}-\xi / B_{s}\right)\left(r_{p}+\xi / \beta_{p}\right)}{\left(d_{s}+\xi / B_{s}\right)\left(r_{p}-\xi / \beta_{p}\right)}, & r<-\frac{\xi}{B_{s}}, \\ -2 \beta_{p} r_{p}-\xi \ln \frac{r_{p}-\xi / \beta_{p}}{r_{p}+\xi / \beta_{p}}, & r>-\frac{\xi}{B_{s}},\end{cases}
$$

where

$$
B=-\frac{F}{8 \pi} \frac{1}{B_{s}^{2}+\beta_{p}^{2}}, \quad d_{s}^{2}=\frac{\xi^{2}}{B_{s}^{2}}-r^{2}, \quad r_{p}^{2}=\frac{\xi^{2}}{\beta_{p}^{2}}+r^{2}
$$

\section{Supersonic penetration}

In the two-dimensional case

$$
\square_{s}^{2} \square_{p}^{2} \widetilde{L}(\xi, y)=F \delta(\xi) \delta(y)
$$

with the condition of no upstream influence, namely $\widetilde{L} \rightarrow 0$ as $\xi^{2}+y^{2} \rightarrow \infty, \xi>0$. Hence, writing $\Psi=\square_{p}^{2} \widetilde{L}, X=\xi / B_{s}$ gives

$$
\square^{2} \Psi(X, y)=F \frac{1}{B_{s}} \delta(X) \delta(y), \quad \square^{2}=-\frac{\partial^{2}}{\partial X^{2}}+\frac{\partial^{2}}{\partial y^{2}}
$$

whose relevant solution is

$$
\Psi=-\frac{F}{4 B_{s}} H(-(X-y)) H(-(X+y)) .
$$

The next step is to solve the equation:

$$
\square_{p}^{2} \widetilde{L}(\xi, y)=-\frac{F}{4 B_{s}} H\left(-\left(\xi / B_{s}-y\right)\right) H\left(-\left(\xi / B_{s}+y\right)\right) .
$$

Hence

$\widetilde{L}_{F}=-\frac{F}{4 B_{s}} \begin{cases}0, & -\frac{3}{4} \pi<\theta<\frac{3}{4} \pi, \\ \frac{\lambda}{1-\lambda^{2}} r^{2}(\cos \theta+\sin \theta)^{2}, & \frac{3}{4} \pi<\theta<\pi-\arctan \lambda, \\ \frac{1}{2(1-\lambda)} r^{2}\left(\lambda \cos ^{2} \theta+\sin ^{2} \theta\right), & \pi-\arctan \lambda<\theta<\pi+\arctan \lambda, \\ \frac{\lambda}{1-\lambda^{2}} r^{2}(\cos \theta+\sin \theta)^{2}, & \pi+\arctan \lambda<\theta<2 \pi-\frac{3}{4} \pi,\end{cases}$

where

$$
r^{2}=\frac{\xi^{2}}{B_{p}^{2}}+y^{2}, \quad \lambda=B_{p} / B_{s}
$$

Article submitted to Royal Society 
and

$$
\theta= \begin{cases}\arctan \frac{B_{p} y}{\xi}, & \xi>0, \quad y>0, \\ \arctan \frac{B_{p} y}{\xi}+\pi, & \xi<0, \\ \arctan \frac{B_{p} y}{\xi}+2 \pi, & \xi>0, \quad y<0 .\end{cases}
$$

Similarly, the Love function satisfies

$$
\square_{s}^{2} \square_{p}^{2} \widetilde{L}(\xi, r)=F \delta(x) \delta(y) \delta(\xi)
$$

with far-field conditions analogous to those for (A 27). We write $\Psi=\square_{p}^{2} \widetilde{L}$ and $\xi=B_{s} Z$ to give

$$
\square^{2} \Psi(Z, r)=F \frac{1}{B_{s}} \delta(x) \delta(y) \delta(Z),
$$

whose relevant solution is

$$
\Psi(Z, r)= \begin{cases}0, & r>-Z, \\ \frac{F}{4 \pi} \frac{1}{B_{s}} \frac{1}{\sqrt{Z^{2}-r^{2}}}, & r<-Z .\end{cases}
$$

Hence we solve

$$
\square_{p}^{2} \widetilde{L}(\xi, r)= \begin{cases}0, & r>-\xi / B_{s}, \\ \frac{F}{4 \pi} \frac{1}{B_{s}} \frac{1}{\sqrt{\xi^{2} / B_{s}^{2}-r^{2}}}, & r<-\xi / B_{s},\end{cases}
$$

which, with $\xi=B_{p} Z$ and $\lambda=B_{p} / B_{s}$, becomes

$$
\square^{2} \widetilde{L}(Z, r)= \begin{cases}0, & r>-\lambda Z, \\ \frac{F}{4 \pi} \frac{1}{B_{s}} \frac{1}{\sqrt{\lambda^{2} Z^{2}-r^{2}}}, & r<-\lambda Z,\end{cases}
$$

with the solution:

$$
\widetilde{L}(Z, r)=F \frac{\alpha_{2}}{\alpha_{1}}\left\{\begin{array}{cc}
\frac{1}{\lambda} \sqrt{\lambda^{2} Z^{2}-r^{2}}-\sqrt{Z^{2}-r^{2}} & r<-\lambda Z, \\
+\frac{1}{2} Z \ln -\frac{\left(\sqrt{\lambda^{2} Z^{2}-r^{2}}-\lambda Z\right)\left(\sqrt{Z^{2}-r^{2}}+Z\right)}{\left(\sqrt{\lambda^{2} Z^{2}-r^{2}}+\lambda Z\right)\left(\sqrt{Z^{2}-r^{2}}-Z\right)}, & \\
-\left(\sqrt{Z^{2}-r^{2}}+\frac{1}{2} Z \ln \frac{\sqrt{Z^{2}-r^{2}}-Z}{\sqrt{Z^{2}-r^{2}}+Z}\right), & r>-\lambda Z,
\end{array}\right.
$$

where

$$
\alpha_{1}=-\frac{1}{2 \mu(1-\nu)} \frac{1}{B_{p}}, \quad \alpha_{2}=\frac{1}{4 \pi \mu} \frac{1}{1+B_{s}^{2}}, \quad \nu=\frac{1}{2}\left(1-\frac{1+B_{p}^{2}}{B_{s}^{2}-B_{p}^{2}}\right) .
$$


In $(\xi, r)$ coordinates the solution becomes:

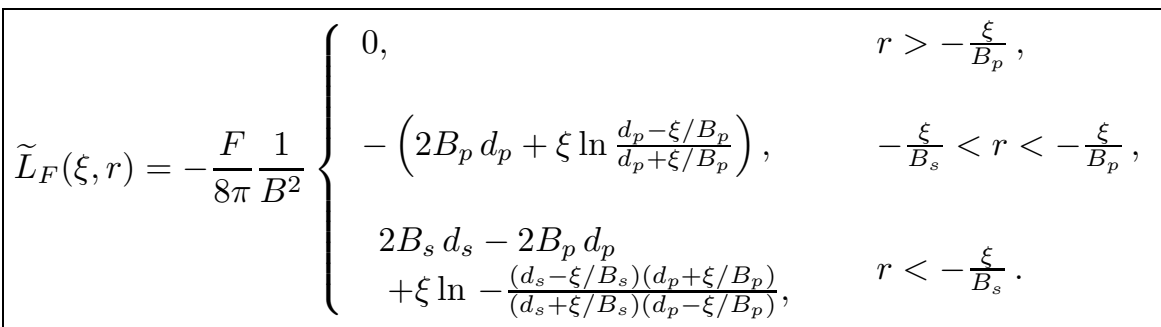

(A 35)

where

$$
B^{2}=B_{s}^{2}-B_{p}^{2}, \quad d_{s}^{2}=\frac{\xi^{2}}{B_{s}^{2}}-r^{2}, \quad d_{p}^{2}=\frac{\xi^{2}}{B_{p}^{2}}-r^{2} .
$$

(ii) Nuclei of strain

Nuclei of strain can be modelled by two pairs of point forces for two-dimensions and by three pairs of point forces for three-dimensions applied at the origin. In two-dimensions the displacements are simply related to the dynamic Love stress function $L$ defined in (A 1) via

$u_{x}=-\frac{1}{2 \mu} \frac{1-2 \nu}{1-\nu} \frac{\partial}{\partial x}\left(\nabla^{2}-\frac{1}{c_{s}^{2}} \frac{\partial^{2}}{\partial t^{2}}\right) L, \quad u_{y}=-\frac{1}{2 \mu} \frac{1-2 \nu}{1-\nu} \frac{\partial}{\partial y}\left(\nabla^{2}-\frac{1}{c_{s}^{2}} \frac{\partial^{2}}{\partial t^{2}}\right) L$.

In axial symmetry the displacements are related to $L$ in a similar way

$u_{r}=-\frac{1}{2 \mu} \frac{1-2 \nu}{1-\nu} \frac{\partial}{\partial r}\left(\nabla^{2}-\frac{1}{c_{s}^{2}} \frac{\partial^{2}}{\partial t^{2}}\right) L, \quad u_{z}=-\frac{1}{2 \mu} \frac{1-2 \nu}{1-\nu} \frac{\partial}{\partial z}\left(\nabla^{2}-\frac{1}{c_{s}^{2}} \frac{\partial^{2}}{\partial t^{2}}\right) L$.

Using these definitions and the boxed expressions for dynamic Love stress functions $L_{F}$ from the previous section one can easily obtain the displacements, strain and stress components for subsonic, transonic and supersonic regimes.

(b) Residual stress region 


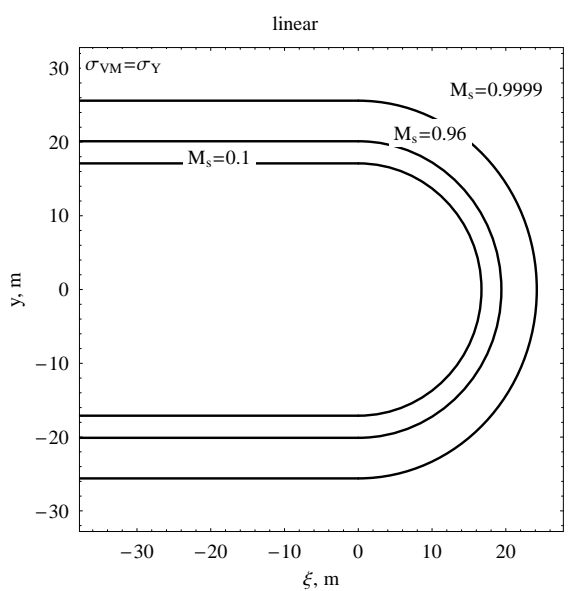

(a)

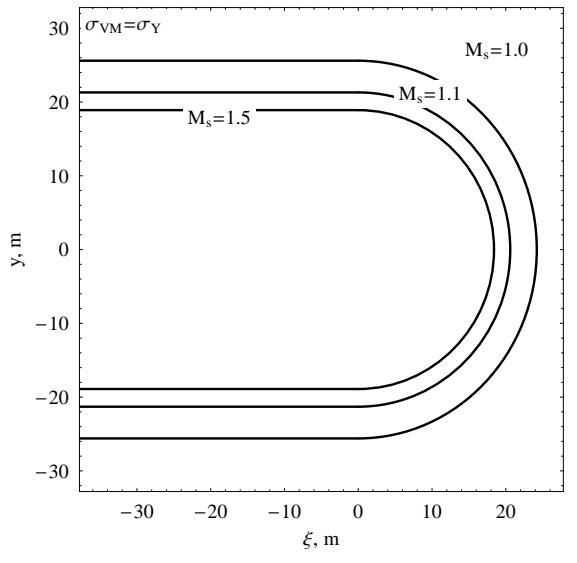

(b)

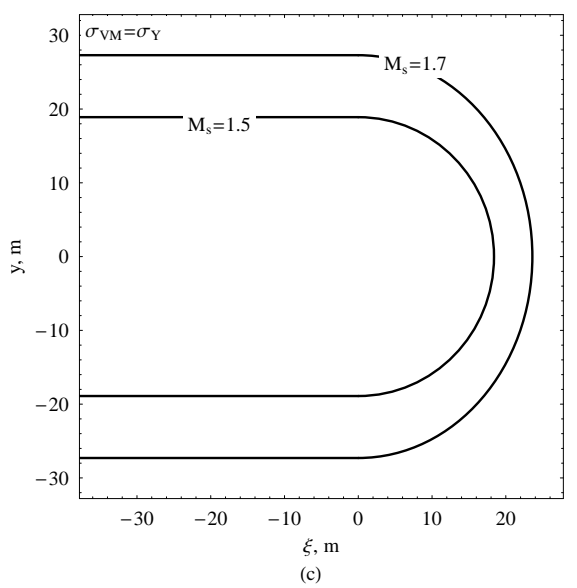

axisymmetric

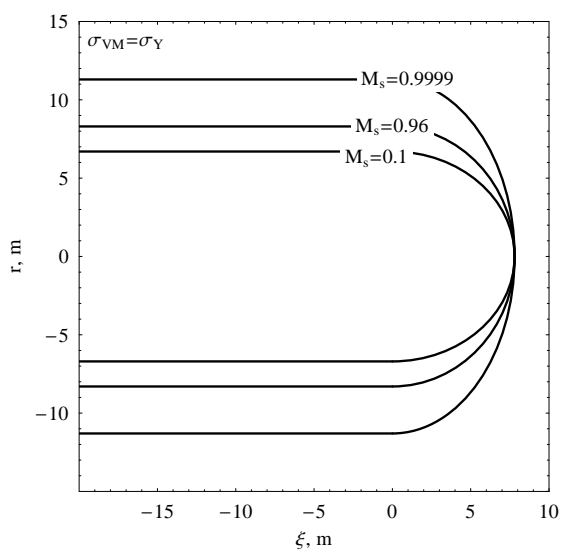

(d)

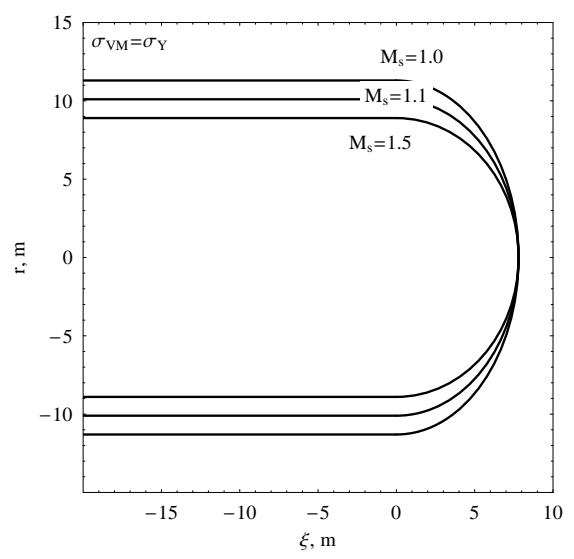

(e)

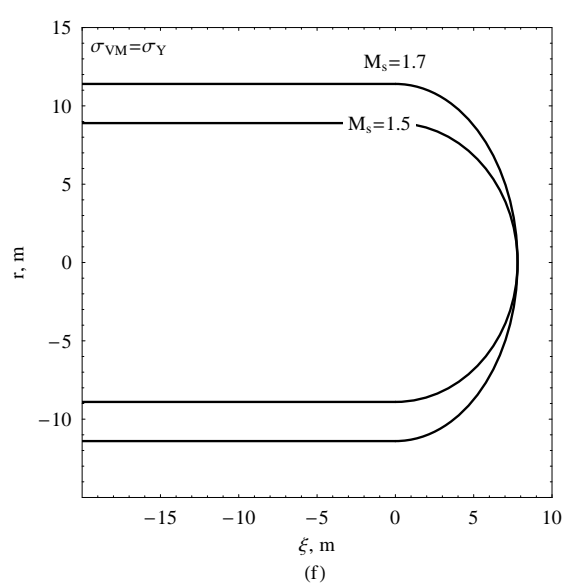

Figure A1. Residual stress regions depending on the Mach number. Curves (a), (d) correspond to subsonic penetration with $M_{s}<1$, (b), (e) to transonic penetration with $1<M_{s}<1.5$, (c), (f) to transonic penetration with $1.5<M_{s}<c_{p} / c_{s}$.

Article submitted to Royal Society 